\title{
Steric and Electronic Effects on Ligand Substitution of Cationic Carbonyl Nitrosyl Complexes of Molybdenum(0) Containing Bis(3,5-dimethylpyrazol-1-yl)methane- $N, N^{\prime}{ }^{1}$
}

\author{
Kom-Bei Shiu, ${ }^{* 2 a}$ Sheng-Ting Lin, ${ }^{2 a}$ Dei-Wei Fung, ${ }^{2 a}$ Tsung-Jung Chan, ${ }^{2 a}$ Shie-Ming Peng, ${ }^{2 b}$ \\ Ming-Chu Cheng, ${ }^{2 b}$ and J. L. Chou ${ }^{2 a}$
}

Department of Chemistry, National Cheng Kung University, Tainan, Taiwan 701, Republic of China, and National Taiwan University, Taipei, Taiwan 107, Republic of China

Received June 3, $1994^{\otimes}$

Nitrosylation of either $\left[\mathrm{M}(\mathrm{N}, \mathrm{N})(\mathrm{CO})_{4}\right]$ or $\left[\mathrm{Mo}\left(\mathrm{H}_{2} \mathrm{CPz}_{2}^{\prime}\right)(\mathrm{CO})_{2}(\mathrm{NO}) \mathrm{I}\right]$, with $\mathrm{NO}^{+} \mathrm{BF}_{4}{ }^{-}$in $\mathrm{MeOH}$, afforded readily $\left[\mathrm{M}(\mathrm{N}, \mathrm{N})(\mathrm{CO})_{2}(\mathrm{NO})(\mathrm{MeOH})\right] \mathrm{BF}_{4}\left(\mathrm{M}=\mathrm{Mo},(\mathrm{N}, \mathrm{N})=\mathrm{H}_{2} \mathrm{CPz}_{2}(\mathbf{1}), \mathrm{H}_{2} \mathrm{CPz}_{2}^{\prime}(2) ; \mathrm{M}=\mathrm{W},(\mathrm{N}, \mathrm{N})=\mathrm{H}_{2} \mathrm{CPz}_{2}(3)\right.$, $\mathrm{H}_{2} \mathrm{CPz}_{2}^{\prime}$ (4); $\mathrm{Pz}=$ pyrazol-1-yl; $\mathrm{Pz}^{\prime}=3,5$-dimethylpyrazol-1-yl). 2 reacted with py to form $\left[\mathrm{Mo}\left(\mathrm{H}_{2} \mathrm{CPz}_{2}^{\prime}\right)\right.$ $\left.(\mathrm{CO})_{2}(\mathrm{NO})(\mathrm{py})\right] \mathrm{BF}_{4}(5)$. Chloride, bromide, iodide, azide, nitrate, thiocyanide, acetate, and cyanide reacted with 2 in $\mathrm{MeOH}$ to form $\left[\mathrm{Mo}\left(\mathrm{H}_{2} \mathrm{CPz}_{2}^{\prime}\right)(\mathrm{CO})_{2}(\mathrm{NO}) \mathrm{L}\right]\left(\mathrm{L}^{-}=\mathrm{Cl}^{-}(6), \mathrm{Br}^{-}(7), \mathrm{I}^{-}(8), \mathrm{N}_{3}^{-}(9), \mathrm{NO}_{3}^{-}(\mathbf{1 0}), \mathrm{SCN}^{-}(11)\right.$, $\left.\mathrm{MeCO}_{2}^{-}(\mathbf{1 2}), \mathrm{CN}^{-}(14)\right)$. Alternatively, 7 could also be prepared from reaction of $\left[\mathrm{Mo}\left(\mathrm{H}_{2} \mathrm{CPz}_{2}{ }_{2}\right)(\mathrm{CO})_{2} \mathrm{Br}_{2}\right]$ with $\mathrm{NaNO}_{2}, n-\mathrm{Bu}_{4} \mathrm{NNO}_{2}$, or $\mathrm{AgNO}_{2}$ in $\mathrm{CH}_{2} \mathrm{Cl}_{2}$ but with a low yield. In the presence of $\mathrm{Cl}^{-}, \mathrm{Br}^{-}$, and $\mathrm{I}^{-}$in $\mathrm{MeOH}$, 5 was transformed back into 6,7 , and 8, respectively. 2 reacted with a 0.5 equiv of $\mathrm{KCN}$ or with 1 equiv of 14 in $\mathrm{MeOH}$ to form $\left[\left\{\mathrm{Mo}_{2}\left(\mathrm{H}_{2} \mathrm{CPz}_{2}^{\prime}\right)(\mathrm{CO})_{2}(\mathrm{NO})\right\}_{2}(\mu-\mathrm{CN})\right](13)$. Both spectral data for 1-14 and X-ray crystallographic structures of 9, 10, and 12 support that 1-4 and 6-14 are similar in geometry around the Mo center with the monodentate ligand ( $\mathrm{MeOH}$ or $\left.\mathrm{L}^{-}\right)$trans to $\mathrm{NO}$ while cis to $\mathrm{H}_{2} \mathrm{CPz}_{2}^{\prime}$ and that 5 may have two conformations with py cis to $\mathrm{H}_{2} \mathrm{CPz}_{2}$ but either trans or cis to NO. 2 reacted with excess $\mathrm{PR}_{3}$ in $\mathrm{CH}_{2} \mathrm{Cl}_{2}$ to form $\left[\mathrm{Mo}\left(\mathrm{H}_{2}-\right.\right.$ $\left.\left.\mathrm{CPz}_{2}^{\prime}\right)(\mathrm{CO})(\mathrm{NO})\left(\mathrm{PR}_{3}\right)_{2}\right] \mathrm{BF}_{4}(\mathrm{R}=\mathrm{Ph}(15), \mathrm{OMe}(16), \mathrm{Me}(17))$. The spectral data for 15-17 and the X-ray crystallographic structure of 17 showed that two phosphine ligands in 15-17 are trans to each other while cis to $\mathrm{H}_{2} \mathrm{CPz}_{2}{ }_{2} .2$ reacted with excess bidentate ligand $(\mathrm{L}, \mathrm{L})$ in $\mathrm{MeOH}$ to form $\left[\mathrm{Mo}(\mathrm{L}, \mathrm{L})_{2}(\mathrm{CO})(\mathrm{NO})\right] \mathrm{BF}_{4}((\mathrm{~L}, \mathrm{~L})=$ bpy (18), phen (19), $\mathrm{Me}_{2}$ bpy (20), dppm (21)). However, when the solvent was changed to $\mathrm{CH}_{2} \mathrm{Cl}_{2}$, several products including 22 were formed. Both ${ }^{1} \mathrm{H}$ and ${ }^{31} \mathrm{P}$ NMR spectral data showed that 18-21 are isostructural with two (L,L) ligands cis to each other. 2 reacted with dithiocarbamate anion (S,S) ${ }^{-}$in $\mathrm{MeCN}$ to form orange-red and yellow products. The orange-red $\left[\mathrm{Mo}(\mathrm{S}, \mathrm{S})(\mathrm{CO})_{2}(\mathrm{NO})\right]_{2}\left((\mathrm{~S}, \mathrm{~S})^{-}=\mathrm{Et}_{2} \mathrm{NCS}_{2}{ }^{-}(\mathbf{2 2}), \mathrm{Me}_{2} \mathrm{NCS}_{2}{ }^{-}(23), \mathrm{C}_{4} \mathrm{H}_{8} \mathrm{NCS}_{2}{ }^{-}\right.$ (24)) as a major product was isolated. As supported by the X-ray crystallographic structure of 22, 22-24 are dimeric with the six-electron (S,S) ${ }^{-}$ligand bonded in a $\mu, \eta^{2}$-fashion. The distance of $3.474(3) \AA$ between two $\mathrm{S}$ atoms and that of 3.773(1) $\AA$ between two Mo atoms is compatible with no S-S or Mo-Mo bond. The nitrosyl groups are trans to the bridging sulfur atoms while $c$ is to carbonyls. Structural data: 9, $a=11.9868(19)$ $\AA, b=10.7998(10) \AA, c=14.723(3) \AA, \beta=113.995(13)^{\circ}, P 2_{1} / c ; 10, a=12.993(4) \AA, b=9.497(3) \AA, c=$ $15.090(5) \AA, \beta=105.49(3)^{\circ}, P 2, c ; 12, a=8.854(7) \AA, b=11.904(7) \AA, c=18.238(7) \AA, \alpha=88.06(4)^{\circ}, \beta$ $=97.65(1)^{\circ}, \gamma=80.83(5)^{\circ}, P \overline{1} ; 17, a=16.637(3) \AA, b=12.379(3) \AA, c=14.6574(20) \AA, \beta=103.284(14)^{\circ}$, $P 2{ }_{1} / c ; 22, a=15.597(4) \AA, b=12.026(3) \AA, c=13.511(3) \AA, \beta=104.271(19)^{\circ}, C 2 / c$.

\section{Introduction}

In order to keep the pyrazolyl ring planar, neutral nitrogen bidentate ligands $(\mathrm{N}, \mathrm{N})$ such as dipyrazol-1-ylmethane- $N, N^{\prime}\left(\mathrm{H}_{2-}\right.$ $\left.\mathrm{CPz}_{2}\right)$ or bis (3,5-dimethylpyrazol-1-yl)diarylmethane- $N, N^{\prime}\left(\mathrm{Ar}_{2}-\right.$ $\mathrm{CPz}_{2}^{\prime}$ ) form six-membered-boat metallacycles naturally when chelating a transition-metal atom (Figure 1). The inherent ring strain present in the metallacycle was previously shown in the facile displacement of $(\mathrm{N}, \mathrm{N})$ of $\left[\mathrm{M}(\mathrm{N}, \mathrm{N})(\mathrm{CO})_{4}\right](\mathrm{M}=\mathrm{Cr}, \mathrm{Mo}$, $\mathrm{W}),{ }^{3}\left[\mathrm{Mo}(\mathrm{N}, \mathrm{N})(\mathrm{CO})_{2}\left(\eta^{3}\right.\right.$-allyl) $\left.\mathrm{Br}\right],{ }^{4-6}$ and $\left[\mathrm{Mo}(\mathrm{N}, \mathrm{N})(\mathrm{CO})_{2} \mathrm{Br}_{2}\right]^{5}$ by $\mathrm{MeCN}, \mathrm{PR}_{3}(\mathrm{R}=\mathrm{Me}, \mathrm{OMe}$ ), bis(diphenylphosphino)methane (dppm), 1,2-bis(diphenylphosphino)ethane (dppe), and dithiocarbamate $\left(\mathbf{S}, \mathbf{S}^{-}{ }^{-}\right.$to afford straightforwardly mononuclear

${ }^{\otimes}$ Abstract published in Advance ACS Abstracts, January 15, 1995.

(1) Dedicated to Professor F. Albert Cotton on the occasion of his 65th birthday and the graduation of his 100 th $\mathrm{Ph} . \mathrm{D}$. student.

(2) (a) National Cheng Kung University. (b) National Taiwan University.

(3) Lobbia, G. G.; Bonati, F. J. Organomet. Chem. 1989, 366, 121.

(4) Shiu, K.-B.; Chang, C.-J.; Wang, S.-L.; Liao, F.-L. J. Organomet. Chem. 1991, 407, 225.

(5) Shiu, K.-B.; Yih, K.-H.; Wang, S.-L.; Liao, F.-L. J. Organomet. Chem. $1991,414,165$

(6) Shiu, K.-B.; Yih, K.-H.; Wang, S.-L.; Liao, F.-L. J. Organomet. Chem. $1991,420,359$ products. However, as described in detail in this paper, reactions of a nitrosyl derivative such as $\left[\mathrm{Mo}\left(\mathrm{H}_{2} \mathrm{CPz}_{2}^{\prime}\right)(\mathrm{CO})_{2}(\mathrm{NO})\right.$ $(\mathrm{MeOH})]^{+}$with a variety of nucleophiles afforded complicated products, which may still contain $\mathrm{H}_{2} \mathrm{CPz}_{2}^{\prime}$ and are either monoor dimeric (Scheme 1).

\section{Experimental Section}

General Procedures. All manipulations were conducted with standard Schlenk techniques. ${ }^{7}$ A blanket of predried dinitrogen was used over each reaction. Solvents were purified by distillation from an appropriate drying agent [ethers, paraffins, and arenes from potassium with benzophenone as indicator; halocarbons and acetonitrile from $\mathrm{CaH}_{2}$; alcohols from the corresponding alkoxide]. ${ }^{1} \mathrm{H}$ and ${ }^{31} \mathrm{P}$ NMR spectra were recorded on Bruker WP-100 ('H, $100 \mathrm{MHz})$, AM$200\left({ }^{\mathrm{H}} \mathrm{H}, 200 \mathrm{MHz}\right), \mathrm{AM}-300\left({ }^{1} \mathrm{H}, 300 \mathrm{MHz}\right)$, and AMC-400 $\left({ }^{1} \mathrm{H}, 400\right.$ $\left.\mathrm{MHz} ;{ }^{31} \mathrm{P}, 162 \mathrm{MHz}\right)$ and Varian Unity Plus-400 $\left({ }^{1} \mathrm{H}, 400 \mathrm{MHz} ;{ }^{31} \mathrm{P}\right.$, $162 \mathrm{MHz})$ NMR spectrometers. ${ }^{1} \mathrm{H}$ chemical shifts $(\delta$ in $\mathrm{ppm}, J$ in $\mathrm{Hz}$ ) are defined as positive downfield relative to internal $\mathrm{MeSi}_{4}$ (TMS) or the deuterated solvent, while ${ }^{31} \mathrm{P}$ chemical shifts are defined as positive downfield relative to external $85 \% \mathrm{H}_{3} \mathrm{PO}_{4}$ or internal free

(7) Shriver, D. F. Manipulation of Air-Sensitive Compounds; McGrawHill: New York, 1969.

(c) 1995 American Chemical Society 
Scheme $1^{a}$

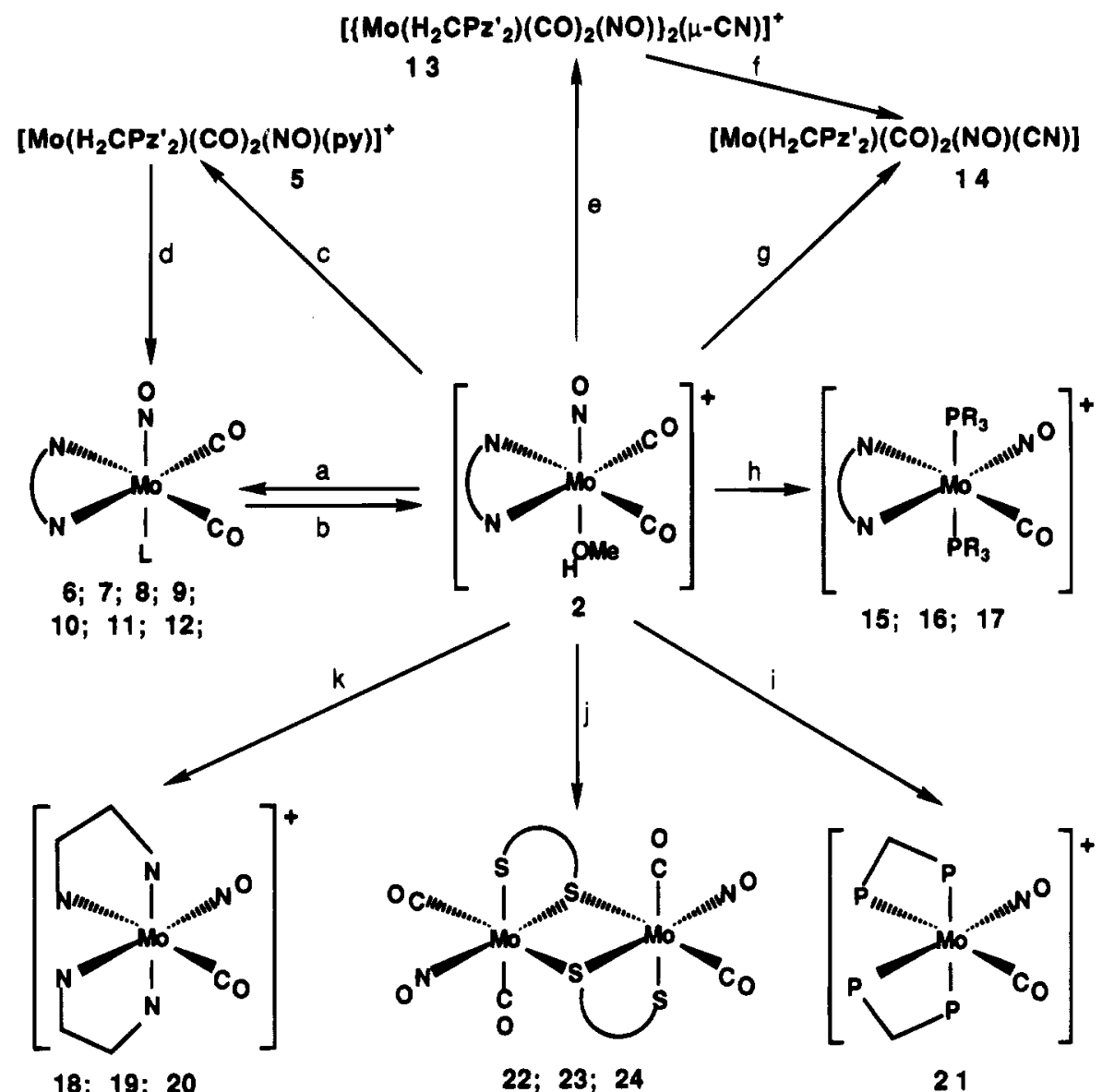

a Key: (a) $\mathrm{NaL}$ or $\mathrm{KL}\left(\mathrm{L}^{-}=\mathrm{Cl}^{-}, \mathrm{I}^{-}, \mathrm{N}_{3}^{-}, \mathrm{NO}_{3}^{-}, \mathrm{SCN}^{-}, \mathrm{MeCO}_{2}^{-}\right.$); (b) for $\mathrm{L}^{-}=\mathrm{I}^{-}, \mathrm{NO}^{+} \mathrm{BF}_{4}^{-}$; (c) py; (d) $\mathrm{NaCl}, \mathrm{NaBr}$ or $\mathrm{NaI}$; (e) 14 or $1 / 2$ $\mathrm{KCN}$; (f) $1 / 2 \mathrm{KCN}$; (g) KCN; (h) $2 \mathrm{PR}_{3}$; (i) $2 \mathrm{dppm}$; (j) $\mathrm{Na}^{+} \mathrm{Et}_{2} \mathrm{NCS}_{2}^{-}, \mathrm{Na}^{+} \mathrm{Me}_{2} \mathrm{NCS}_{2}^{-}$or $\mathrm{NH}_{4}{ }^{+} \mathrm{C}_{4} \mathrm{H}_{8} \mathrm{NCS}_{2}^{-}$; (k) bpy, phen or $\mathrm{Me}_{2}$ bpy.

phosphine ligand with $\delta\left({ }^{31} \mathrm{P}\right)=141$ for $\mathrm{P}(\mathrm{OMe})_{3},-61$ for $\mathrm{PMe}_{3},{ }^{8 \mathrm{a}}-6$ for $\mathrm{PPh}_{3},{ }^{8 b}$ and -23.6 for $\mathrm{dppm}^{8 \mathrm{c}}$ (s, singlet; $\mathrm{d}$, doublet; m, multiplet; br, broad). Infrared spectra were recorded by using a Hitachi 260 - or 270-30 instrument (vs, very strong; s, strong; m, medium; $w$, weak; br, broad; sh, shoulder). Elemental analysis results were obtained by the staff of the Microanalytical Service of the Department of Chemistry, National Cheng Kung University. The complexes $\left[\mathrm{M}(\mathrm{N}, \mathrm{N})(\mathrm{CO})_{4}\right](\mathrm{M}$ $=\mathrm{Mo}, \mathrm{W} ;(\mathrm{N}, \mathrm{N})=\mathrm{H}_{2} \mathrm{CPz}_{2}$ or $\left.\mathrm{H}_{2} \mathrm{CPz}_{2}^{\prime}\right)^{9}$ and $\left[\mathrm{Mo}\left(\mathrm{H}_{2} \mathrm{CPz}_{2}{ }_{2}\right)(\mathrm{CO})_{2} \mathrm{Br}_{2}\right]^{10}$ were prepared according to the literature methods.

Preparation of $\left[\mathrm{Mo}\left(\mathrm{H}_{2} \mathrm{CPz}_{2}\right)(\mathrm{CO})_{2}(\mathrm{NO})(\mathrm{MeOH})\right] \mathrm{BF}_{4}(1)$. To a stirred suspension of $\left[\mathrm{Mo}\left(\mathrm{H}_{2} \mathrm{CPz}_{2}\right)(\mathrm{CO})_{4}\right](0.358 \mathrm{~g}, 1.0 \mathrm{mmol})$ in $\mathrm{MeOH}$ $(20 \mathrm{~mL})$ was added [NO]BF $4(0.234 \mathrm{~g}, 2.0 \mathrm{mmol})$ in portions. Gas evolved briskly, and the suspension soon became a clear orange-yellow solution. When evolution of gas ceased, the volume of the solution was reduced to $c a .2 \mathrm{~mL}$ and $\mathrm{Et}_{2} \mathrm{O}(15 \mathrm{~mL})$ was added to give an orange-yellow precipitate, collected on a medium frit. Yield: $0.40 \mathrm{~g}$ (89\%). IR $\left(\mathrm{CH}_{2} \mathrm{Cl}_{2}\right): v_{\mathrm{CO}}, 2044 \mathrm{~s}, 1964 \mathrm{~s} ; v_{\mathrm{NO}}, 1660 \mathrm{~s} \mathrm{~cm}^{-1}$. 'H NMR $\left(298 \mathrm{~K}, \mathrm{CD}_{3} \mathrm{OD}, 100 \mathrm{MHz}\right): \mathrm{MeOH}, \delta 3.36(3 \mathrm{H}, \mathrm{s}) ; \mathrm{CH}_{2}, 6.49(1 \mathrm{H}$, d, $\left.{ }^{2} J_{\mathrm{H}, \mathrm{H}}=15\right), 6.97\left(1 \mathrm{H}, \mathrm{d},{ }^{2} J_{\mathrm{H}, \mathrm{H}}=15\right)$; hydrogen atoms on the ring-4 position $\left(\mathrm{H}_{(4)}\right) 6.67(2 \mathrm{H}, \mathrm{t})$; hydrogen atoms on the ring-3 and -5 positions $\left(\mathrm{H}_{(3)}, \mathrm{H}_{(5)}\right), 8.15\left(2 \mathrm{H}, \mathrm{d},{ }^{3} J_{\mathrm{H}, \mathrm{H}}=2.3\right), 8.30\left(2 \mathrm{H}, \mathrm{d},{ }^{3} J_{\mathrm{H}, \mathrm{H}}=\right.$ 2.5). Anal. Calcd for $\mathrm{C}_{10} \mathrm{H}_{12} \mathrm{BF}_{4} \mathrm{MoN}_{5} \mathrm{O}_{4}: \mathrm{C}, 26.75 ; \mathrm{H}, 2.69 ; \mathrm{N}, 15.60$. Found: $\mathrm{C}, 26.85 ; \mathrm{H}, 2.75 ; \mathrm{N}, 15.55$.

(8) (a) Mathieu, R.; Lenzi, M.; Poilblanc, R. Inorg. Chem. 1970, 9, 2030. (b) Grim, S. O.; Wheatland, D. A.; McFarlane, W. J. Am. Chem. Soc. 1967, 89, 5572. (c) Horn, H. G.; Sommer, K. Spectrochim. Acta 1971, $27 \mathrm{~A}, 1049$.

(9) (a) Shiu, K.-B.; Chang, C.-J. J. Chin. Chem. Soc. (Taipei) 1987, 34. 297. (b) Shiu, K.-B.; Liou, K.-S.; Wang, Y.; Cheng, M.-C.; Lee, G.-H. J. Organomet. Chem. 1993, 453, 201.

(10) (a) Shiu, K.-B.; Liou, K.-S.; Wang, S.-L.; Cheng, C. P.; Wu, F.-J. J. Organomet. Chem. 1989, 359, C1. (b) Shiu, K.-B.; Liou, K.-S.; Wang, S.-L.; Wei, S.-C. Organometallics 1990, 9, 669.
Preparation of $\left[\mathrm{Mo}\left(\mathrm{H}_{2} \mathrm{CPz}_{2}^{\prime}\right)(\mathrm{CO})_{2}(\mathrm{NO})(\mathrm{MeOH})\right] \mathrm{BF}_{4}(2)$. This orange-yellow compound was prepared similarly from $\left[\mathrm{Mo}\left(\mathrm{H}_{2}-\right.\right.$ $\left.\left.\mathrm{CPz}^{\prime}{ }_{2}\right)(\mathrm{CO})_{4}\right]$ in $91 \%$ yield. IR $\left(\mathrm{CH}_{2} \mathrm{Cl}_{2}\right): v_{\mathrm{CO}}, 2040 \mathrm{~s}, 1960 \mathrm{~s} ; v_{\mathrm{NO}}$, $1659 \mathrm{~s} \mathrm{~cm}^{-1}$. ${ }^{1} \mathrm{H}$ NMR $\left(298 \mathrm{~K}, \mathrm{CDCl}_{3}, 100 \mathrm{MHz}\right)$ : methyl groups on the ring-3 and -5 positions $\left(\mathrm{Me}_{(3)}, \mathrm{Me}_{(5)}\right), \delta 2.45(\mathrm{~s}), 2.47(\mathrm{~s}), 2.50$ (s); $\mathrm{MeOH}, 3.48(3 \mathrm{H}, \mathrm{s}), 1.60(1 \mathrm{H}, \mathrm{s}) ; \mathrm{H}_{(4)}, 6.04(\mathrm{~s}), 6.07(\mathrm{~s}) ; \mathrm{CH}_{2}, 6.15$ (br), $6.32\left(\mathrm{~d},{ }^{2} J_{\mathrm{H}, \mathrm{H}}=16\right), 6.57\left(\mathrm{~d},{ }^{2} J_{\mathrm{H}, \mathrm{H}}=16\right)$. Anal. Calcd for $\mathrm{C}_{14} \mathrm{H}_{20} \mathrm{BF}_{4} \mathrm{MoN}_{5} \mathrm{O}_{4}:$ C, $33.29 ; \mathrm{H}, 3.99 ; \mathrm{N}, 13.87$. Found: C, 32.97 ; $\mathrm{H}, 4.16 ; \mathrm{N}, 13.65$.

Preparation of $\left[\mathrm{W}\left(\mathrm{H}_{2} \mathrm{CPz}_{2}\right)(\mathrm{CO})_{2}(\mathrm{NO})(\mathrm{MeOH})\right] \mathrm{BF}_{4}(3)$. This orange-yellow compound was obtained by a procedure similar to that of 1 from $\left[\mathrm{W}\left(\mathrm{H}_{2} \mathrm{CPz}_{2}\right)(\mathrm{CO})_{4}\right]$ in $60 \%$ yield. IR $\left(\mathrm{CH}_{3} \mathrm{CN}\right): v_{\mathrm{CO}}, 2010$ $\mathrm{s}, 1902 \mathrm{~s} ; v_{\mathrm{NO}}, 1632 \mathrm{~s} \mathrm{~cm}^{-1}$. ' $\mathrm{H}$ NMR $\left(298 \mathrm{~K}, \mathrm{CD}_{3} \mathrm{CN}, 100 \mathrm{MHz}\right)$ : $\mathrm{MeOH}, \delta 3.28(3 \mathrm{H}, \mathrm{s}), 2.09(1 \mathrm{H}, \mathrm{s}) ; \mathrm{CH}_{2}, 6.39\left(1 \mathrm{H}, \mathrm{d},{ }^{2} J_{\mathrm{H}, \mathrm{H}}=15\right)$, $6.74\left(1 \mathrm{H}, \mathrm{d},{ }^{2} J_{\mathrm{H}, \mathrm{H}}=15\right) ; \mathrm{H}_{(4)}, 6.61(2 \mathrm{H}, \mathrm{t}) ; \mathrm{H}_{(3)}, \mathrm{H}_{(5)}, 8.12(2 \mathrm{H}, \mathrm{d}$, $\left.{ }^{3} J_{\mathrm{H}, \mathrm{H}}=2.1\right), 8.17\left(2 \mathrm{H}, \mathrm{d},{ }^{3} J_{\mathrm{H}, \mathrm{H}}=2.7\right)$. Anal. Caled for $\mathrm{C}_{10} \mathrm{H}_{12-}$ $\mathrm{BF}_{4} \mathrm{~N}_{5} \mathrm{O}_{4} \mathrm{~W}$ : C, $22.37 ; \mathrm{H}, 2.25 ; \mathrm{N}, 13.05$. Found: $\mathrm{C}, 22.17 ; \mathrm{H}, 2.33$; $\mathrm{N}, 12.96$

Preparation of $\left[\mathrm{W}\left(\mathrm{H}_{2} \mathrm{CPz}_{2}{ }_{2}\right)(\mathrm{CO})_{2}(\mathrm{NO})(\mathrm{MeOH})\right] \mathrm{BF}_{4}$ (4). This orange-yellow compound was prepared similarly from $\left[\mathrm{W}\left(\mathrm{H}_{2} \mathrm{CPz}_{2}^{\prime}\right)(\mathrm{CO})_{4}\right]$ in $72 \%$ yield. IR $\left(\mathrm{CH}_{2} \mathrm{Cl}_{2}\right): v_{\mathrm{CO}}, 2028 \mathrm{~s}, 1928 \mathrm{~s} ; v_{\mathrm{NO}}, 1644 \mathrm{~s} \mathrm{~cm}^{-1} .{ }^{1} \mathrm{H}$ NMR $\left(298 \mathrm{~K}, \mathrm{CDCl}_{3}, 200 \mathrm{MHz}\right): \mathrm{Me}_{(3)}, \mathrm{Me}_{(5)}, \delta 2.43(6 \mathrm{H}, \mathrm{br}), 2.47$ $(6 \mathrm{H}, \mathrm{br})$; $\mathrm{MeOH}, 3.28(3 \mathrm{H}, \mathrm{s}), 1.60(1 \mathrm{H}, \mathrm{s}) ; \mathrm{H}_{(4)}, 6.25(2 \mathrm{H}, \mathrm{br})$; $\mathrm{CH}_{2}, 6.16\left(1 \mathrm{H}, \mathrm{d},{ }^{2} J_{\mathrm{H}, \mathrm{H}}=16\right), 6.28\left(1 \mathrm{H}, \mathrm{d},{ }^{2} J_{\mathrm{H}, \mathrm{H}}=16\right)$. Anal. Calcd for $\mathrm{C}_{14} \mathrm{H}_{20} \mathrm{BF}_{4} \mathrm{~N}_{5} \mathrm{O}_{4} \mathrm{~W}$ : C, $28.35 ; \mathrm{H}, 3.40 ; \mathrm{N}, 11.81$. Found: C, 28.50; $\mathrm{H}, 3.42 ; \mathrm{N}, 11.53$.

Preparation of $\left[\mathrm{Mo}\left(\mathrm{H}_{2} \mathrm{CPz}_{2}{ }_{2}\right)(\mathrm{CO})_{2}(\mathrm{NO})(\mathrm{py})\right] \mathrm{BF}_{4}(5)$. To a stirred solution of $2(0.253 \mathrm{~g}, 0.50 \mathrm{mmol})$ in $\mathrm{CH}_{2} \mathrm{Cl}_{2}(25 \mathrm{~mL})$ was added pyridine (py) ( $1 \mathrm{~mL}, \mathrm{ca} .12 \mathrm{mmol})$. The solution was stirred for 10 min, and the solvent was removed under vacuum. Recrystallization from $\mathrm{CH}_{2} \mathrm{Cl}_{2}$ /hexane gave the yellow product $(0.25 \mathrm{~g}, 90 \%)$. IR $\left(\mathrm{CH}_{2}-\right.$ $\left.\mathrm{Cl}_{2}\right): v_{\mathrm{CO}}, 2036 \mathrm{~s}, 1948 \mathrm{~s} ; v_{\mathrm{NO}}, 1680 \mathrm{~s}, 1657 \mathrm{sh} \mathrm{cm}^{-1}$. 'H NMR (298 $\mathrm{K}$, acetone- $d_{6}, 200 \mathrm{MHz}$ ): $\mathrm{Me}_{(3)}, \mathrm{Me}_{(5)}, \delta 2.47(\mathrm{~s}), 2.58(\mathrm{~s}), 2.62$ (s), 
$2.67(\mathrm{~s}) ; \mathrm{H}_{(4)}$ or $\mathrm{CH}_{2}, 6.19-6.70(\mathrm{~m}) ; \mathrm{py}, 7.57(2 \mathrm{H}, \mathrm{m}), 8.16(2 \mathrm{H}, \mathrm{m})$, $8.74(1 \mathrm{H}, \mathrm{br})$. Anal. Calcd for $\mathrm{C}_{18} \mathrm{H}_{21} \mathrm{BF}_{4} \mathrm{MoN}_{6} \mathrm{O}_{3}: \mathrm{C}, 39.16 ; \mathrm{H}, 3.83$; $\mathrm{N}, 15.22$. Found: C, 38.93; $\mathrm{H}, 3.79 ; \mathrm{N}, 15.15$.

Preparation of $\left[\mathrm{Mo}\left(\mathrm{H}_{2} \mathrm{CPz}_{2}{ }_{2}\right)(\mathrm{CO})_{2}(\mathrm{NO}) \mathrm{Cl}\right]$ (6). To a stirred solution of $2(0.253 \mathrm{~g}, 0.50 \mathrm{mmol})$ in $\mathrm{MeOH}(20 \mathrm{~mL})$ was added $\mathrm{NaCl}$ $(0.030 \mathrm{~g}, 0.75 \mathrm{mmol})$. The solution was stirred for $1.5 \mathrm{~h}$, forming a yellow precipitate. The solvent was removed under vacuum. Recrystallization from $\mathrm{CH}_{2} \mathrm{Cl}_{2} / \mathrm{MeOH}$ gave the yellow product $(0.19 \mathrm{~g}, 91 \%)$. IR $\left(\mathrm{CH}_{2} \mathrm{Cl}_{2}\right): v_{\mathrm{CO}}, 2032 \mathrm{~s}, 1944 \mathrm{~s} ; v_{\mathrm{NO}}, 1634 \mathrm{~s} \mathrm{~cm}^{-1}$. 'H NMR (298 $\mathrm{K}$, acetone- $\left.d_{6}, 200 \mathrm{MHz}\right): \mathrm{Me}_{(3)}, \mathrm{Me}_{(5)}, \delta 2.52(6 \mathrm{H}, \mathrm{s}), 2.58(6 \mathrm{H}, \mathrm{s})$; $\mathrm{H}_{(4)}, 6.20(2 \mathrm{H}, \mathrm{br}) ; \mathrm{CH}_{2}, 6.30(1 \mathrm{H}, \mathrm{br}), 6.38(1 \mathrm{H}, \mathrm{br})$. Anal. Calcd for $\mathrm{C}_{13} \mathrm{H}_{16} \mathrm{ClMoN}_{5} \mathrm{O}_{3}$ : C, 37.03; H, 3.82; N, 16.61. Found: C, 37.06; $\mathrm{H}, 3.83 ; \mathrm{N}, 16.54$

Compound 6 was also formed as a yellow precipitate from the reaction between 5 and excess $\mathrm{NaCl}$ dissolved in $\mathrm{MeOH}$.

Preparation of $\left[\mathrm{Mo}\left(\mathrm{H}_{2} \mathrm{CPz}_{2}^{\prime}\right)(\mathrm{CO})_{2}(\mathrm{NO}) \mathrm{Br}\right]$ (7). This yellow compound was prepared in $94 \%$ yield by a procedure analogous to that of 6 , either from the reaction between 2 and $\mathrm{NaBr}$ or that between 5 and this salt. IR $\left(\mathrm{CH}_{2} \mathrm{Cl}_{2}\right): v_{\mathrm{CO}}, 2032 \mathrm{~s}, 1944 \mathrm{~s} ; v_{\mathrm{NO}}, 1640 \mathrm{~s} \mathrm{~cm}^{-1}$. ${ }^{1} \mathrm{H}$ NMR $\left(298 \mathrm{~K}\right.$, acetone- $\left.d_{6}, 200 \mathrm{MHz}\right): \mathrm{Me}_{(3)}, \mathrm{Me}_{(5)}, \delta 2.55(6 \mathrm{H}, \mathrm{s})$, $2.60(6 \mathrm{H}, \mathrm{s}) ; \mathrm{H}_{(4)}, 6.22(2 \mathrm{H}, \mathrm{br}) ; \mathrm{CH}_{2}, 6.27(1 \mathrm{H}, \mathrm{br}), 6.42(1 \mathrm{H}, \mathrm{br})$ Anal. Calcd for $\mathrm{C}_{13} \mathrm{H}_{16} \mathrm{BrMoN}_{5} \mathrm{O}_{3} ; \mathrm{C}, 33.50 ; \mathrm{H}, 3.46 ; \mathrm{N}, 15.02$. Found: $\mathrm{C}, 33.53 ; \mathrm{H}, 3.52 ; \mathrm{N}, 14.87$

Compound 7 was also obtained by adding $\mathrm{NaNO}_{2}, n-\mathrm{Bu}_{4} \mathrm{NNO}_{2}$, or $\mathrm{AgNO}_{2}$ to $\left[\mathrm{Mo}\left(\mathrm{H}_{2} \mathrm{CPz}_{2}\right)(\mathrm{CO})_{2} \mathrm{Br}_{2}\right]$ at a yield $33-36 \%$ : To a stirred solution of $\left[\mathrm{Mo}\left(\mathrm{H}_{2} \mathrm{CPz}_{2}{ }_{2}\right)(\mathrm{CO})_{2} \mathrm{Br}_{2}\right](0.516 \mathrm{~g}, 1.00 \mathrm{mmol})$ in $\mathrm{CH}_{2} \mathrm{Cl}_{2}$ $(20 \mathrm{~mL})$ was added $\mathrm{NaNO}_{2}, n-\mathrm{Bu}_{4} \mathrm{NNO}_{2}$, or $\mathrm{AgNO}_{2}(1.00 \mathrm{mmol})$, dissolved in $\mathrm{MeOH}(5 \mathrm{~mL})$. The solution was stirred for $10 \mathrm{~min}$. The solvent was then removed under vacuum. Recrystallization from $\mathrm{CH}_{2-}$ $\mathrm{Cl}_{2} / \mathrm{MeOH}$ gave a yellow product.

Preparation of $\left[\mathrm{Mo}\left(\mathrm{H}_{2} \mathrm{CPz}_{2}^{\prime}\right)(\mathrm{CO})_{2}(\mathrm{NO}) \mathrm{I}\right](8)$. This orange-yellow compound was prepared in $94 \%$ yield by a procedure analogous to that of 6 , either from the reaction between 2 and $\mathrm{NaI}$ or that between 5 and this salt. IR $\left(\mathrm{CH}_{2} \mathrm{Cl}_{2}\right): v_{\mathrm{CO}}, 2028 \mathrm{~s}, 1944 \mathrm{~s} ; v_{\mathrm{NO}}, 1642 \mathrm{~s} \mathrm{~cm}^{-1}$. ${ }^{1} \mathrm{H}$ NMR $\left(298 \mathrm{~K}\right.$, acetone- $\left.d_{6}, 200 \mathrm{MHz}\right): \mathrm{Me}_{(3)}, \mathrm{Me}_{(5)}, \delta 2.56(6 \mathrm{H}, \mathrm{s})$, $2.62(6 \mathrm{H}, \mathrm{s}) ; \mathrm{H}_{(4)}, 6.24(2 \mathrm{H}, \mathrm{br}) ; \mathrm{CH}_{2}, 6.33(1 \mathrm{H}, \mathrm{br}), 6.40(1 \mathrm{H}, \mathrm{br})$. Anal. Calcd for $\mathrm{C}_{13} \mathrm{H}_{16} \mathrm{IMoN}_{5} \mathrm{O}_{3}: \mathrm{C}, 30.37 ; \mathrm{H}, 3.14 ; \mathrm{N}, 13.65$. Found: C, 30.43; H, 3.13; N, 13.32 .

Preparation of $\left[\mathrm{Mo}\left(\mathrm{H}_{2} \mathrm{CPz}_{2}{ }_{2}\right)(\mathrm{CO})_{2}(\mathrm{NO})\left(\eta^{1}-\mathrm{N}_{3}\right)\right](9)$. This yellow compound was prepared in $87 \%$ yield by a procedure analogous to that of 6, from 2 and $\mathrm{NaN}_{3}$. IR $\left(\mathrm{CH}_{2} \mathrm{Cl}_{2}\right): v_{\mathrm{CO}}, 2024 \mathrm{~s}, 1938 \mathrm{~s} ; v_{\mathrm{NNN}}$, $2076 \mathrm{~s} ; \nu_{\mathrm{NO}}, 1634 \mathrm{~s} \mathrm{~cm}^{-1}$. 'H NMR $\left(298 \mathrm{~K}\right.$, acetone- $\left.d_{6}, 200 \mathrm{MHz}\right)$ : $\mathrm{Me}_{(3)}, \mathrm{Me}_{(5)}, \delta 2.48(6 \mathrm{H}, \mathrm{s}), 2.58(6 \mathrm{H}, \mathrm{s}) ; \mathrm{H}_{(4)}, 6.21(2 \mathrm{H}, \mathrm{br}) ; \mathrm{CH}_{2}$, $6.30(1 \mathrm{H}$, br $), 6.38\left(1 \mathrm{H}\right.$, br). Anal. Caled for $\mathrm{C}_{13} \mathrm{H}_{16} \mathrm{MoN}_{8} \mathrm{O}_{3}$ : C, 36.46; H, 3.77; N, 26.16. Found: C, 36.39; H, 3.78; N, 26.15.

Caution! Although we have experienced no explosions, azides must always be treated with great care.

Preparation of $\left[\mathrm{Mo}\left(\mathrm{H}_{2} \mathrm{CPz}_{2}\right)(\mathrm{CO})_{2}(\mathrm{NO})\left(\eta^{1}-\mathrm{NO}_{3}\right)\right](\mathbf{1 0})$. This yellow compound was prepared in $82 \%$ yield by a procedure analogous to that of 9, using $\mathrm{NaNO}_{3}$. IR $\left(\mathrm{CH}_{2} \mathrm{Cl}_{2}\right): v_{\mathrm{CO}}, 2036 \mathrm{~s}, 1948 \mathrm{~s} ; v_{\mathrm{NO}}$, $1630 \mathrm{~s} \mathrm{~cm}^{-1}$. ' $\mathrm{H}$ NMR $\left(298 \mathrm{~K}\right.$, acetone- $\left.d_{6}, 200 \mathrm{MHz}\right): \mathrm{Me}_{(3)}, \mathrm{Me}_{(5)}$, $\delta 2.37(6 \mathrm{H}, \mathrm{s}), 2.60(6 \mathrm{H}, \mathrm{s}) ; \mathrm{H}_{(4)}, 6.28(2 \mathrm{H}, \mathrm{br}) ; \mathrm{CH}_{2}, 6.30(1 \mathrm{H}, \mathrm{d}$, $J=16), 6.67(1 \mathrm{H}, \mathrm{d}, J=16)$. Anal. Calcd for $\mathrm{C}_{15} \mathrm{H}_{19} \mathrm{MoN}_{5} \mathrm{O}_{5}: \mathrm{C}$, 34.83; H, 3.60; N, 18.75. Found: C, 34.56; H, 3.55; N, 18.38.

Preparation of $\left[\mathrm{Mo}\left(\mathrm{H}_{2} \mathrm{CPz}_{2}^{\prime}\right)(\mathrm{CO})_{2}(\mathrm{NO})(\mathrm{SCN})\right](11)$. This yellow compound was prepared in $95 \%$ yield by a procedure analogous to that of 9, using KSCN. IR $\left(\mathrm{CH}_{2} \mathrm{Cl}_{2}\right): v_{\mathrm{CO}}, 2032 \mathrm{~s}, 1946 \mathrm{~s} ; v_{\mathrm{SCN}}, 2072$ s; $v_{\mathrm{NO}}, 1646 \mathrm{~s} \mathrm{~cm}^{-1}$. 'H NMR (acetone- $d_{6}, 300 \mathrm{MHz}$ ): $\mathrm{Me}_{(3)}, \mathrm{Me}_{(5)}, \delta$ $2.46(6 \mathrm{H}, \mathrm{s}), 2.60(6 \mathrm{H}, \mathrm{s}) ; \mathrm{H}_{(4)}, 6.23(2 \mathrm{H}, \mathrm{br}) ; \mathrm{CH}_{2}, 6.38(1 \mathrm{H}, \mathrm{br})$, $6.65(1 \mathrm{H}, \mathrm{d}, J=16)$ at $298 \mathrm{~K}$ and $\mathrm{Me}_{(3)}, \mathrm{Me}_{(5)}, \delta 2.47(6 \mathrm{H}, \mathrm{s}), 2.60$ $(6 \mathrm{H}, \mathrm{s}) ; \mathrm{H}_{(4)}, 6.21(2 \mathrm{H}, \mathrm{br}) ; \mathrm{CH}_{2}, 6.38(1 \mathrm{H}, \mathrm{d}, J=16), 6.65(1 \mathrm{H}, \mathrm{d}$, $J=16$ ) at $328 \mathrm{~K}$. Anal. Calcd for $\mathrm{C}_{14} \mathrm{H}_{16} \mathrm{MoN}_{6} \mathrm{O}_{3} \mathrm{~S}: \mathrm{C}, 37.84 ; \mathrm{H}$, $3.63 ; \mathrm{N}, 18.91$. Found: $\mathrm{C}, 37.77 ; \mathrm{H}, 3.68 ; \mathrm{N}, 18.85$.

Preparation of $\left[\mathrm{Mo}\left(\mathrm{H}_{2} \mathrm{CPz}_{2}{ }_{2}\right)(\mathrm{CO})_{2}(\mathrm{NO})\left(\eta^{1}-\mathrm{MeCO}_{2}\right)\right](12)$. This yellow compound was prepared in $88 \%$ yield by a procedure analogous to that of 9, using sodium acetate, $\mathrm{NaMeCO}_{2}$. IR $\left(\mathrm{CH}_{2} \mathrm{Cl}_{2}\right): v_{\mathrm{CO}}, 2032$ s, $1932 \mathrm{~s} ; v_{\mathrm{NO}}, 1630 \mathrm{~s} \mathrm{~cm}^{-1}$. 'H NMR (298 K, acetone- $\left.d_{6}, 200 \mathrm{MHz}\right)$ : $\mathrm{Me}_{(3)}, \mathrm{Me}_{(5)}, \delta 2.34$ (6 H, s), $2.45(6 \mathrm{H}, \mathrm{s})$; OAC, $2.57(3 \mathrm{H}, \mathrm{s}) ; \mathrm{H}_{(4)}$, $6.17(2 \mathrm{H}, \mathrm{br}) ; \mathrm{CH}_{2}, 6.34(1 \mathrm{H}, \mathrm{br}), 6.41(1 \mathrm{H}, \mathrm{br})$. Anal. Calcd for $\mathrm{C}_{15} \mathrm{H}_{19} \mathrm{MoN}_{5} \mathrm{O}_{5}: \mathrm{C}, 40.46 ; \mathrm{H}, 4.30 ; \mathrm{N}, 15.73$. Found: $\mathrm{C}, 40.39 ; \mathrm{H}$, $4.28 ; \mathrm{N}, 15.53$.
Preparation of $\left[\left\{\mathrm{Mo}\left(\mathrm{H}_{2} \mathrm{CPz}_{2}^{\prime}\right)(\mathrm{CO})_{2}(\mathrm{NO})\right\}_{2}(\mu-\mathrm{CN})\right] \mathrm{BF}_{4}(13)$ and $\left[\mathrm{Mo}\left(\mathrm{H}_{2} \mathrm{CPz}_{2}^{\prime}\right)(\mathrm{CO})_{2}(\mathrm{NO})(\mathrm{CN})\right](14)$. To a stirred solution of $2(0.505$ $\mathrm{g}, 1.00 \mathrm{mmol})$ in $\mathrm{MeOH}(20 \mathrm{~mL})$ was added a solution of KCN $(0.065$ $\mathrm{g}, 1.00 \mathrm{mmol})$, dissolved in $\mathrm{MeOH}(10 \mathrm{~mL})$, dropwise. An orangeyellow precipitate was formed first on addition of half the $\mathrm{KCN}$ solution, but the solution became clear after complete addition. The precipitate was isolated by filtration after addition of half the $\mathrm{KCN}$ solution and characterized as 13. Yield, 63\%. IR $\left(\mathrm{CH}_{2} \mathrm{Cl}_{2}\right): v_{\mathrm{CN}}, 2140 \mathrm{w} ; v_{\mathrm{CO}}, 2040$ s, $1952 \mathrm{~s} ; v_{\mathrm{NO}}, 1668 \mathrm{~s} \mathrm{~cm}^{-1}$. 'H NMR $\left(298 \mathrm{~K}\right.$, acetone- $\left.d_{6}, 200 \mathrm{MHz}\right)$ : $\mathrm{Me}_{(3)}, \mathrm{Me}_{(5)}, \delta 1.93(6 \mathrm{H}, \mathrm{s}), 1.98(6 \mathrm{H}, \mathrm{s}), 2.65(12 \mathrm{H}, \mathrm{br}) ; \mathrm{H}_{(4)}, 6.19$ $(2 \mathrm{H}, \mathrm{br}), 6.22(2 \mathrm{H}, \mathrm{br}) ; \mathrm{CH}_{2}, 6.31(1 \mathrm{H}, \mathrm{br}), 6.37(1 \mathrm{H}, \mathrm{br}), 6.68(1$ $\mathrm{H}, \mathrm{br}), 6.76\left(1 \mathrm{H}\right.$, br). Anal. Calcd for $\mathrm{C}_{27} \mathrm{H}_{32} \mathrm{BF}_{4} \mathrm{Mo}_{2} \mathrm{~N}_{11} \mathrm{O}_{6}$ : C, 36.63; $\mathrm{H}, 3.64 ; \mathrm{N}, 17.40$. Found: $\mathrm{C}, 36.64 ; \mathrm{H}, 3.70 ; \mathrm{N}, 17.44$. In another preparation, after complete addition of the $\mathrm{KCN}$ solution, the $\mathrm{MeOH}$ was stripped from the clear orange-yellow solution under vacuum. The product, 14, was recrystallized from $\mathrm{CH}_{2} \mathrm{Cl}_{2} / \mathrm{Et}_{2} \mathrm{O}$ to give $0.30 \mathrm{~g}(73 \%)$. IR $\left(\mathrm{CH}_{2} \mathrm{Cl}_{2}\right): v_{\mathrm{CN}}, 2116 \mathrm{w} ; v_{\mathrm{CO}}, 2032 \mathrm{~s}, 1940 \mathrm{~s} ; v_{\mathrm{NO}}, 1654 \mathrm{~s} \mathrm{~cm}{ }^{-1} .{ }^{1} \mathrm{H}$ NMR $\left(298 \mathrm{~K}\right.$, acetone- $\left.d_{6}, 200 \mathrm{MHz}\right): \mathrm{Me}_{(3)}, \mathrm{Me}_{(5)}, \delta 2.48(6 \mathrm{H}, \mathrm{s})$, $2.60(6 \mathrm{H}, \mathrm{s}) ; \mathrm{H}_{(4)}, 6.23(2 \mathrm{H}, \mathrm{br}) ; \mathrm{CH}_{2}, 6.39(1 \mathrm{H}, \mathrm{br}), 6.46(1 \mathrm{H}, \mathrm{br})$. Anal. Calcd for $\mathrm{C}_{14} \mathrm{H}_{16} \mathrm{MoN}_{6} \mathrm{O}_{3}: \mathrm{C}, 40.79 ; \mathrm{H}, 3.91 ; \mathrm{N}, 20.39$. Found: $\mathrm{C}, 40.69 ; \mathrm{H}, 3.81 ; \mathrm{N}, 20.15$. Altematively, 13 could also be prepared from 2 and 1 equiv of 14.

Preparation of $\left[\mathrm{Mo}\left(\mathrm{H}_{2} \mathrm{CPz}_{2}{ }_{2}\right)(\mathrm{CO})(\mathrm{NO})\left(\mathrm{PPh}_{3}\right)_{2}\right] \mathrm{BF}_{4}(\mathbf{1 5})$. To a stirred solution of $2(0.258 \mathrm{~g}, 0.51 \mathrm{mmol})$ in $\mathrm{CH}_{2} \mathrm{Cl}_{2}(25 \mathrm{~mL})$ was added $\mathrm{PPh}_{3}(0.278 \mathrm{~g}, 1.06 \mathrm{mmol})$. The solution was heated under reflux for $2 \mathrm{~h}$, and the solvent was removed under vacuum. The solid residue was then washed with $\mathrm{Et}_{2} \mathrm{O}(15 \mathrm{~mL})$ to remove the unreacted $\mathrm{PPh}_{3}$. Recrystallization from $\mathrm{CH}_{2} \mathrm{Cl}_{2} /$ hexane gave the orange-red product $(0.47$ $\mathrm{g}, 95 \%)$. The compound is slightly air-sensitive and should be stored under $\mathrm{N}_{2}$. IR $\left(\mathrm{CH}_{2} \mathrm{Cl}_{2}\right): v_{\mathrm{CO}}, 1922 \mathrm{~s} ; v_{\mathrm{NO}}, 1640 \mathrm{~s} \mathrm{~cm}^{-1}$. 'H NMR $\left(298 \mathrm{~K}\right.$, acetone- $\left.d_{6}, 200 \mathrm{MHz}\right): \mathrm{Me}_{(3)}, \mathrm{Me}_{(5)}, \delta 2.29(6 \mathrm{H}, \mathrm{m}), 2.53(6$ $\mathrm{H}, \mathrm{m}) ; \mathrm{H}_{(4)}, 5.61(2 \mathrm{H}, \mathrm{m}) ; \mathrm{CH}_{2}, 6.23(\mathrm{~m}), 6.79(\mathrm{~m}) .{ }^{31} \mathrm{P}\left\{{ }^{1} \mathrm{H}\right\} \mathrm{NMR}$ $\left(298 \mathrm{~K}, \mathrm{CH}_{2} \mathrm{Cl}_{2} /\right.$ acetone- $\left.d_{6}=1 / 1,162 \mathrm{MHz}\right): \delta 46.5(\mathrm{br})$. Anal. Calcd for $\mathrm{C}_{48} \mathrm{H}_{46} \mathrm{BF}_{4} \mathrm{MoN}_{5} \mathrm{O}_{2} \mathrm{P}_{2}: \mathrm{C}, 59.64 ; \mathrm{H}, 4.78 ; \mathrm{N}, 7.22$. Found: $\mathrm{C}, 59.42$; $\mathrm{H}, 4.82 ; \mathrm{N}, 7.24$.

Preparation of $\left[\mathrm{Mo}\left(\mathrm{H}_{2} \mathrm{CPz}_{2}{ }_{2}\right)(\mathrm{CO})(\mathrm{NO})\left\{\mathrm{P}(\mathrm{OMe})_{3}\right\}_{2}\right] \mathrm{BF}_{4}(16)$. To a stirred solution of $2(0.267 \mathrm{~g}, 0.53 \mathrm{mmol})$ in $\mathrm{CH}_{2} \mathrm{Cl}_{2}(25 \mathrm{~mL})$ was added $\mathrm{P}(\mathrm{OMe})_{3}(0.44 \mathrm{~mL}, 3.7 \mathrm{mmol})$. The solution was heated under reflux for $44 \mathrm{~h}$, and the solvent was removed under vacuum. Recrystallization from $\mathrm{CH}_{2} \mathrm{Cl}_{2}$ /hexane gave the orange-yellow product $(0.32 \mathrm{~g}, 88 \%)$. IR $\left(\mathrm{CH}_{2} \mathrm{Cl}_{2}\right): v_{\mathrm{CO}}, 1948 \mathrm{~s} ; v_{\mathrm{NO}}, 1660 \mathrm{~s} \mathrm{~cm}^{-1}$. ' $\mathrm{H}$ NMR $\left(298 \mathrm{~K}\right.$, acetone- $\left.d_{6}, 200 \mathrm{MHz}\right): \mathrm{Me}_{(3)}, \mathrm{Me}_{(5)}, \delta 2.54(6 \mathrm{H}, \mathrm{d}, J=11)$, $2.58(6 \mathrm{H}, \mathrm{d}, J=11) ; \mathrm{P}(\mathrm{OMe})_{3}, 3.49(18 \mathrm{H}, J=6) ; \mathrm{H}_{(4)}, 6.33(2 \mathrm{H}$, br); $\mathrm{CH}_{2}, 6.27(1 \mathrm{H}, \mathrm{br}), 6.36(1 \mathrm{H}, \mathrm{br}),{ }^{3 !} \mathrm{P}\left\{{ }^{1} \mathrm{H}\right\} \mathrm{NMR}\left(298 \mathrm{~K}, \mathrm{CH}_{2}-\right.$ $\mathrm{Cl}_{2} /$ acetone $-d_{6}=1 / 1,162 \mathrm{MHz}$ ): $\delta 152.2(\mathrm{br})$. Anal. Calcd for $\mathrm{C}_{18} \mathrm{H}_{32} \mathrm{BF}_{4} \mathrm{MoN}_{5} \mathrm{O}_{8} \mathrm{P}_{2}: \mathrm{C}, 31.19 ; \mathrm{H}, 4.94 ; \mathrm{N}, 10.10$. Found: $\mathrm{C}, 31.15$; H, 4.86; N, 10.13 .

Preparation of $\left[\mathrm{Mo}\left(\mathrm{H}_{2} \mathrm{CPz}_{2}{ }_{2}\right)(\mathrm{CO})(\mathrm{NO})\left(\mathrm{PMe}_{3}\right)_{2}\right] \mathrm{BF}_{4}(17)$. This red-brown compound was prepared in $89 \%$ yield by a procedure analogous to that of 16, using excess $\mathrm{PMe}_{3}(1.0 \mathrm{M}$ solution in THF) and 2. The reaction was completed in $2 \mathrm{~h}$. IR $\left(\mathrm{CH}_{2} \mathrm{Cl}_{2}\right): v_{\mathrm{CO}}, 1914 \mathrm{~s}$; $v_{\mathrm{NO}}, 1634 \mathrm{~s} \mathrm{~cm}^{-1}$. ${ }^{1} \mathrm{H}$ NMR $\left(298 \mathrm{~K}\right.$, acetone- $\left.d_{6}, 200 \mathrm{MHz}\right): \mathrm{Me}_{(3)}$, $\mathrm{Me}_{(5)}, \delta 2.56(6 \mathrm{H}, \mathrm{d}, J=9), 2.59(6 \mathrm{H}, \mathrm{d}, J=9) ; \mathrm{PMe}_{3}, 1.24(18 \mathrm{H}$, t, $J=3) ; \mathrm{H}_{(4)}, 5.99(2 \mathrm{H}, \mathrm{br}) ; \mathrm{CH}_{2}, 6.31(1 \mathrm{H}, \mathrm{br}), 6.38(1 \mathrm{H}, \mathrm{br})$. ${ }^{31} \mathrm{P}\left\{{ }^{1} \mathrm{H}\right\}$ NMR $\left(298 \mathrm{~K}, \mathrm{CH}_{2} \mathrm{Cl}_{2} /\right.$ acetone- $\left.d_{6}=1 / 1,162 \mathrm{MHz}\right): \delta-5.9$ (br). Anal. Calcd for $\mathrm{C}_{18} \mathrm{H}_{34} \mathrm{BF}_{4} \mathrm{MoN}_{5} \mathrm{O}_{2} \mathrm{P}_{2}: \mathrm{C}, 36.20 ; \mathrm{H}, 5.73 ; \mathrm{N}$, 10.73. Found: C, $36.11 ; \mathrm{H}, 5.51 ; \mathrm{N}, 11.80$.

Preparation of cis-[Mo(bpy $)_{2}(\mathrm{CO})\left(\mathrm{NO}_{3}\right] \mathrm{BF}_{4}(\mathbf{1 8})$. To a stirred solution of $2(0.253 \mathrm{~g}, 0.50 \mathrm{mmol})$ in $\mathrm{CH}_{2} \mathrm{Cl}_{2}(20 \mathrm{~mL})$ was added bpy $(0.199 \mathrm{~g}, 1.28 \mathrm{mmol})$. Upon addition, the yellow transparent solution changed color almost immediately to dark green and then (ca. $2 \mathrm{~h}$ ) became cloudy. After $80 \mathrm{~h}$, the volume of the solution was reduced to ca. $2 \mathrm{~mL}$ and $\mathrm{Et}_{2} \mathrm{O}(10 \mathrm{~mL})$ was added to complete precipitation of the dark green compound while dissolving the excess bpy and the extruded $\mathrm{H}_{2} \mathrm{CPz}_{2}^{\prime}$. Filtration through a medium frit gave $0.21 \mathrm{~g}(81 \%)$. IR $\left(\mathrm{CH}_{2} \mathrm{Cl}_{2}\right): v_{\mathrm{CO}}, 1910 \mathrm{~s} ; v_{\mathrm{NO}}, 1614 \mathrm{~s} \mathrm{~cm}^{-1}$. ${ }^{1} \mathrm{H}$ NMR $\left(25^{\circ} \mathrm{C}\right.$, acetone$\left.d_{6}, 200 \mathrm{MHz}\right): \delta 9.43\left(1 \mathrm{H}, \mathrm{d},{ }^{3} J_{\mathrm{H}, \mathrm{H}}=5.7\right), 9.32\left(1 \mathrm{H}, \mathrm{d},{ }^{3} J_{\mathrm{H}, \mathrm{H}}=6.9\right)$, $8.87\left(1 \mathrm{H}, \mathrm{d},{ }^{3} J_{\mathrm{H}, \mathrm{H}}=8.2\right), 8.73\left(2 \mathrm{H}, \mathrm{d},{ }^{3} J_{\mathrm{H}, \mathrm{H}}=7.7\right), 8.70\left(1 \mathrm{H}, \mathrm{d},{ }^{3} J_{\mathrm{H}, \mathrm{H}}\right.$ $=6.8), 8.51(1 \mathrm{H}, \mathrm{m}), 8.21(3 \mathrm{H}, \mathrm{m}), 8.01(1 \mathrm{H}, \mathrm{m}), 7.77(3 \mathrm{H}, \mathrm{m})$, $7.52(2 \mathrm{H}, \mathrm{m})$. Anal. Calcd for $\mathrm{C}_{21} \mathrm{H}_{16} \mathrm{BF}_{4} \mathrm{MoN}_{5} \mathrm{O}_{2}: \mathrm{C}, 45.60 ; \mathrm{H}, 2.92$; $\mathrm{N}, 12.66$. Found: $\mathrm{C}, 45.43 ; \mathrm{H}, 3.02 ; \mathrm{N}, 12.67$.

Alternatively, this compound was prepared in a shorter period by 
Table 1. Crystal Data for Mono- and Dinuclear Nitrosyl Complexes

\begin{tabular}{|c|c|c|c|}
\hline & 9 & 17 & 22 \\
\hline formula & $\mathrm{C}_{13} \mathrm{H}_{16} \mathrm{MoN}_{8} \mathrm{O}_{3}$ & $\mathrm{C}_{18} \mathrm{H}_{34} \mathrm{BF}_{4} \mathrm{MoN}_{5} \mathrm{O}_{2} \mathrm{P}_{2}$ & $\mathrm{C}_{14} \mathrm{H}_{20} \mathrm{Mo}_{2} \mathrm{~N}_{4} \mathrm{O}_{6} \mathrm{~S}_{4}$ \\
\hline$M_{\mathrm{r}}$ & 428.26 & 597.18 & 660.44 \\
\hline space group & $P 2_{1} / n$ (No. 14) & $P 2_{1} / c$ (No. 14$)$ & $C 2 / c$ (No. 15) \\
\hline$a, \AA$ & $11.9868(19)$ & $16.637(3)$ & $15.597(4)$ \\
\hline$b, \AA$ & $10.7998(10)$ & $12.379(3)$ & $12.026(3)$ \\
\hline$c, \AA$ & $14.723(3)$ & $14.6574(20)$ & $13.511(3)$ \\
\hline$\alpha, \operatorname{deg}$ & 90 & 90 & 90 \\
\hline$\beta, \mathrm{deg}$ & $113.995(13)$ & $103.284(14)$ & $104.271(19)$ \\
\hline$\gamma, \operatorname{deg}$ & 90 & 90 & 90 \\
\hline$V, \AA^{3}$ & $1741.3(5)$ & $2937.9(10)$ & $2456.2(10)$ \\
\hline$z$ & 4 & 4 & 4 \\
\hline$D_{\text {calc }}, \mathrm{g} \mathrm{cm}^{-3}$ & 1.634 & 1.350 & 1.786 \\
\hline$\mu, \mathrm{mm}^{-1}$ & 0.7638 & 0.5881 & 1.36 \\
\hline no. of reflns colled & 2274 & 3833 & 2161 \\
\hline no. of indep reflns & 2274 & 3830 & 2161 \\
\hline no. of obsd reflns $(>2 \sigma)$ & 1869 & 2415 & 1934 \\
\hline$R^{a}{ }^{a} R_{\mathrm{w}}^{a}$ & $0.022,0.019$ & $0.052,0.049$ & $0.031,0.037$ \\
\hline
\end{tabular}

heating the mixture of $2(0.260 \mathrm{~g}, 0.51 \mathrm{mmol})$ and bpy $(0.200 \mathrm{~g}, 1.28$ $\mathrm{mmol}$ ) in $\mathrm{MeOH}(20 \mathrm{~mL})$ at $65^{\circ} \mathrm{C}$ for $16 \mathrm{~h}$. (The solution changed color from yellow to yellow-brown and then to a dark green suspension within $10 \mathrm{~min}$.) The volume of the suspension was reduced to $c a .3$ $\mathrm{mL}$, and $\mathrm{Et}_{2} \mathrm{O}(15 \mathrm{~mL})$ was added. Filtration gave the dark green solid, which was then dried under vacuum. Yield: $0.22 \mathrm{~g}(79 \%)$.

Preparation of cis-[Mo(phen $)_{2}(\mathrm{CO})(\mathrm{NO}) \mathrm{BF}_{4}(19)$. This dark green compound was obtained by a procedure similar to that of 18 . A typical yield of $0.27 \mathrm{~g}(89 \%)$ was obtained from $2(0.26 \mathrm{~g}, 0.51 \mathrm{mmol})$ and phen $(0.28 \mathrm{~g}, 1.55 \mathrm{mmol})$ in $\mathrm{CH}_{2} \mathrm{Cl}_{2}(20 \mathrm{~mL})$ for $30 \mathrm{~h}$. IR $\left(\mathrm{CH}_{2-}\right.$ $\left.\mathrm{Cl}_{2}\right): v_{\mathrm{CO}}, 1914 \mathrm{~s}, 1934 \mathrm{sh} ; v_{\mathrm{NO}}, 1625 \mathrm{br} \mathrm{cm}^{-1}$. ${ }^{1} \mathrm{H}$ NMR $\left(25{ }^{\circ} \mathrm{C}\right.$ acetone- $\left.d_{6}, 200 \mathrm{MHz}\right): \delta 9.92(1 \mathrm{H}, \mathrm{m}), 9.82(1 \mathrm{H}, \mathrm{m}), 9.16(1 \mathrm{H}, \mathrm{m})$, $8.77(3 \mathrm{H}, \mathrm{m}), 8.40(3 \mathrm{H}, \mathrm{m}), 8.25(3 \mathrm{H}, \mathrm{m}), 8.02(2 \mathrm{H}, \mathrm{m}), 7.64(2 \mathrm{H}$, m). Anal. Calcd for $\mathrm{C}_{25} \mathrm{H}_{16} \mathrm{BF}_{4} \mathrm{MoN}_{5} \mathrm{O}_{2}: \mathrm{C}, 49.95 ; \mathrm{H}, 2.68 ; \mathrm{N}, 11.65$. Found: $\mathrm{C}, 49.62 ; \mathrm{H}, 2.42 ; \mathrm{N}, 11.57$.

Preparation of $c i s-\left[\mathrm{Mo}\left(\mathrm{Me}_{2} \mathrm{bpy}\right)_{2}(\mathrm{CO})(\mathrm{NO})\right] \mathrm{BF}_{4}(\mathbf{2 0})$. This dark green compound was obtained by a procedure similar to that of 18 , by heating the mixture of $2(0.27 \mathrm{~g}, 0.53 \mathrm{mmol})$ and $\mathrm{Me}_{2}$ bpy $(0.22 \mathrm{~g}$, $1.17 \mathrm{mmol})$ in $\mathrm{MeOH}(20 \mathrm{~mL})$ at $65^{\circ} \mathrm{C}$ for $56 \mathrm{~h}$. A typical yield of $0.24 \mathrm{~g}(75 \%)$ was obtained. IR $\left(\mathrm{CH}_{2} \mathrm{Cl}_{2}\right): v_{\mathrm{CO}}, 1904 \mathrm{~s} ; v_{\mathrm{NO}}, 1600 \mathrm{~m}$ $\mathrm{cm}^{-1}$. ${ }^{1} \mathrm{H}$ NMR $\left(25^{\circ} \mathrm{C}\right.$, acetone- $\left.d_{6}, 200 \mathrm{MHz}\right): \delta 9.11(1 \mathrm{H}, \mathrm{d}, J=$ 5.8), $9.08(1 \mathrm{H}, \mathrm{d}, J=5.8), 8.43(1 \mathrm{H}, \mathrm{br}), 8.28(1 \mathrm{H}, \mathrm{br}), 8.20(1 \mathrm{H}$, br), $8.13(1 \mathrm{H}, \mathrm{br}), 7.26(1 \mathrm{H}, \mathrm{d}, J=5.8), 7.30(1 \mathrm{H}, \mathrm{d}, J=5.8), 7.14$ $(4 \mathrm{H}, \mathrm{m}), 2.71(3 \mathrm{H}, \mathrm{s}), 2.65(3 \mathrm{H}, \mathrm{s}), 2.44(3 \mathrm{H}, \mathrm{s}), 2.43(3 \mathrm{H}, \mathrm{s})$. Anal. Calcd for $\mathrm{C}_{25} \mathrm{H}_{24} \mathrm{BF}_{4} \mathrm{MoN}_{5} \mathrm{O}_{2}: \mathrm{C}, 49.28 ; \mathrm{H}, 3.98 ; \mathrm{N}, 11.50$. Found: $\mathrm{C}, 48.90 ; \mathrm{H}, 3.92 ; \mathrm{N}, 11.47$.

Preparation of $c i s-\left[\mathrm{Mo}(\mathrm{dppm})_{2}(\mathrm{CO})(\mathrm{NO})\right] \mathrm{BF}_{4}(\mathbf{2 1})$. This orangered compound was obtained as precipitate by a procedure similar to that of 18 , by heating the mixture of $2(0.23 \mathrm{~g}, 0.45 \mathrm{mmol})$ and dppm $(0.38 \mathrm{~g}, 1.00 \mathrm{mmol})$ in $20 \mathrm{~mL}$ of $\mathrm{MeOH}$ at $65^{\circ} \mathrm{C}$ for $2 \mathrm{~h}$. A typical yield of $0.39 \mathrm{~g}(86 \%)$ was obtained. IR $\left(\mathrm{CH}_{2} \mathrm{Cl}_{2}\right): v_{\mathrm{CO}}, 1964 \mathrm{~s} ; v_{\mathrm{NO}}$ $1650 \mathrm{~m} \mathrm{~cm}^{-1}$. ${ }^{1} \mathrm{H}\left\{{ }^{31} \mathrm{P}\right\}$ NMR $\left(25{ }^{\circ} \mathrm{C}, \mathrm{CH}_{2} \mathrm{Cl}_{2} / \mathrm{CDCl}_{3}=1 / 1,400\right.$ MHz): $\delta 7.85-6.34(40 \mathrm{H}, \mathrm{m}), 4.71(1 \mathrm{H}, \mathrm{d}, J=15), 4.44(1 \mathrm{H}, \mathrm{d}, J$ $=15), 4.35(1 \mathrm{H}, \mathrm{d}, J=15), 3.82(1 \mathrm{H}, \mathrm{d}, J=15) .{ }^{31} \mathrm{P}\left\{{ }^{1} \mathrm{H}\right\} \mathrm{NMR}$ $\left(298 \mathrm{~K}, \mathrm{CH}_{2} \mathrm{Cl}_{2} /\right.$ acetone- $\left.d_{6}=1 / 1,162 \mathrm{MHz}\right): \delta 9.54(1 \mathrm{P}$, ddd, $J=$ $21,27,88), 4.63(1 \mathrm{P}, \mathrm{ddd}, J=16,27,88),-7.25$ (1 P, dt, $J=16$, 27 ), -17.75 ( $1 \mathrm{P}, \mathrm{dt}, J=21,27$ ). Anal. Calcd for $\mathrm{C}_{51} \mathrm{H}_{44} \mathrm{BF}_{4-}$ $\mathrm{MoNO}_{2} \mathrm{P}_{4}: \mathrm{C}, 60.68 ; \mathrm{H}, 4.39 ; \mathrm{N}, 1.39$. Found: $\mathrm{C}, 60.61 ; \mathrm{H}, 4.42 ; \mathrm{N}$, 1.34 .

Preparation of $\left[\mathrm{Mo}\left(\mathrm{Et}_{2} \mathrm{NCS}_{2}\right)(\mathrm{CO})_{2}(\mathrm{NO})\right]_{2}$ (22). A solution of 2 ( $3 \mathrm{mmol}$ ) in $\mathrm{MeCN}(10 \mathrm{~mL}$ ) was added dropwise with a solution of $\mathrm{Na}^{+} \mathrm{Et}_{2} \mathrm{NCS}_{2}{ }^{-}(3 \mathrm{mmol})$ in $\mathrm{MeCN}(30 \mathrm{~mL})$. After addition, the solution was stirred for $30 \mathrm{~min}$ and the solvent was removed under vacuum to give an off-yellow solid residue. The residue was first washed with $\mathrm{Et}_{2} \mathrm{O}(3 \times 20 \mathrm{~mL})$ to remove $\mathrm{H}_{2} \mathrm{CPz}_{2}^{\prime}$ and then dissolved in $\mathrm{CH}_{2} \mathrm{Cl}_{2}$ $(20 \mathrm{~mL})$. Hexane $(60 \mathrm{~mL})$ was carefully poured down the side of the tube onto the solution. After $24 \mathrm{~h}$, many orange-red microcrystals of 22 and some yellow precipitate appeared on the bottom of the flask. The clear solution was removed into another flask through a cannula, and the crystals were washed gently with $\mathrm{CH}_{2} \mathrm{Cl}_{2}(1-2 \mathrm{~mL})$ to dissolve any deposited yellow solid. The resulting yellow solution was also removed by the cannula. Collection of the crystals gave $0.99 \mathrm{~g}$. Yield: 65\%. IR: $v_{\mathrm{CO}}, 2028$ (sh), 2024 (s), 1952 (s), 1936 (s); $v_{\mathrm{NO}}$, 1662 (s) and $v_{\mathrm{CN}}, 1512(\mathrm{~s}) \mathrm{cm}^{-1}$ in $\mathrm{CH}_{2} \mathrm{Cl}_{2} ; v_{\mathrm{CO}}, 2024$ (s), 1936 (s) and $v_{\mathrm{NO}}, 1652(\mathrm{~s}) \mathrm{cm}^{-1}$ in $\mathrm{CH}_{3} \mathrm{CN}$. ${ }^{1} \mathrm{H}$ NMR $(296 \mathrm{~K}, 200 \mathrm{MHz}): \delta 3.86$ $(8 \mathrm{H}, \mathrm{m}), 1.35(12 \mathrm{H}, \mathrm{m})$ in $\mathrm{CDCl}_{3}$ and $\delta 3.86(8 \mathrm{H}, \mathrm{q}, J=7), 1.25(\mathrm{t}$, $12 \mathrm{H}$ ) in acetone- $d_{6}$. Anal. Calcd for $\mathrm{C}_{14} \mathrm{H}_{20} \mathrm{Mo}_{2} \mathrm{~N}_{4} \mathrm{O}_{6} \mathrm{~S}_{4}$ : C, 25.26; $\mathrm{H}, 3.05 ; \mathrm{N}, 8.48$. Found: $\mathrm{C}, 25.44 ; \mathrm{H}, 3.02 ; \mathrm{N}, 8.38$.

Preparation of $\left[\mathrm{Mo}\left(\mathrm{Me}_{2} \mathrm{NCS}_{2}\right)(\mathrm{CO})_{2}(\mathrm{NO})\right]_{2}(23)$. This orange-red compound was prepared by a procedure similar to that of 22 , using $\mathrm{Na}^{+} \mathrm{Me}_{2} \mathrm{NCS}_{2}{ }^{-}$. Yield: 58\%. IR $\left(\mathrm{CH}_{2} \mathrm{Cl}_{2}\right): v_{\mathrm{CO}}, 2026(\mathrm{sh}), 2024(\mathrm{~s})$, $1936(\mathrm{~s}), 1916(\mathrm{~s}) ; v_{\mathrm{NO}}, 1662(\mathrm{~s})$ and $v_{\mathrm{CN}}, 1538(\mathrm{~s}) \mathrm{cm}^{-1}$. ${ }^{1} \mathrm{H}$ NMR (acetone- $\left.d_{6}, 296 \mathrm{~K}, 200 \mathrm{MHz}\right): \delta 3.36(6 \mathrm{H}, \mathrm{s})$. Anal. Calcd for $\mathrm{C}_{10} \mathrm{H}_{14} \mathrm{Mo}_{2} \mathrm{~N}_{4} \mathrm{O}_{6} \mathrm{~S}_{4}: \mathrm{C}, 19.88 ; \mathrm{H}, 1.99 ; \mathrm{N}, 9.27$. Found: C, 19.82; H, $2.10 ; \mathrm{N}, 9.25$.

Preparation of $\left[\mathrm{Mo}\left(\mathrm{C}_{4} \mathrm{H}_{8} \mathrm{NCS}_{2}\right)(\mathrm{CO})_{2}(\mathrm{NO})\right]_{2}$ (24). This orangered compound was prepared by a procedure similar to that of 22, using ammonium pyrrolidinedithiocarbamate $\left(\mathrm{NH}_{4}{ }^{+} \mathrm{Me}_{2} \mathrm{NCS}_{2}{ }^{-}\right)$. Yield: 62\%. IR $\left(\mathrm{CH}_{2} \mathrm{Cl}_{2}\right): v_{\mathrm{CO}}, 2024(\mathrm{~s}), 2014(\mathrm{sh}), 1932$ (s); $v_{\mathrm{NO}}, 1652$ (s) and $v_{\mathrm{CN}}, 1508(\mathrm{~s}) \mathrm{cm}^{-1}$. ${ }^{1} \mathrm{H}$ NMR (acetone- $\left.d_{6}, 296 \mathrm{~K}, 200 \mathrm{MHz}\right): \delta$ $3.70(16 \mathrm{H}, \mathrm{m})$. Anal. Calcd for $\mathrm{C}_{14} \mathrm{H}_{16} \mathrm{Mo}_{2} \mathrm{~N}_{4} \mathrm{O}_{6} \mathrm{~S}_{4}: \mathrm{C}, 25.62 ; \mathrm{H}$, 2.46; N, 8.53. Found: C, 25.64; H, 2.49; N, 8.51.

$\mathrm{X}$-ray Diffraction Measurements. Single crystals were grown by the liquid diffusion method from $\mathrm{CH}_{2} \mathrm{Cl}_{2} /$ hexane, $\mathrm{MeOH} / \mathrm{Et}_{2} \mathrm{O}$, or $\mathrm{MeCN} / \mathrm{Et}_{2} \mathrm{O}$. Diffraction data for $9,10,12,17$, and 22 were collected on a Nonius CAD4 diffractometer with Mo K $\alpha$ radiation $(\lambda=0.70930$ A).

The metal atom in any structure was first solved by the heavy-atom method. The remaining non-hydrogen atoms were subsequently located from the Fourier difference maps, and all atoms except the disordered atom were then refined anisotropically. $(C(5)$ (occupancy $=0.75)$ and $\mathrm{C}\left(5^{*}\right)$ (occupancy $=0.25$ ) in structure 22 were found to be disordered.) The ambiguities of locating $\mathrm{NO}$ and $\mathrm{CO}$ positions, if there are any, were solved according to the chemical and crystallographic meaningfulness of thermal displacement coefficients. A more restricted $\mathrm{N}$ atomic peak with a lower thermal parameter, $U$, than the neighboring $O$ atomic peak confirmed the successful assignment of the NO positions. ${ }^{11}$ Accordingly, only one nitrosyl position was found for any of the five structures described here. The positions of all hydrogen atoms were calculated and refined isotropically to give residual $R$ and $R_{\mathrm{w}}$ values shown in Table 1 with other crystallographic information. All calculations were performed by use of the NRCVAX system of computer programs. ${ }^{12}$

There are two independent molecules, 12A and 12B, of 12 found in the asymmetric unit of the crystal used. Since 9,10 , and $12 \mathrm{~A}$ (or 12B)are isostructural with $\eta^{1}$-azide, -nitrate, and -acetate trans to NO, respectively, only the ORTEP plots for 9 (Figure 2), 17 (Figure 3), and 22 (Figure 4) containing 50\% probability thermal ellipsoids are

(11) Liu, L.-K.; Lin, J.-T.; Fang, D. Inorg. Chim. Acta 1989, 161, 239.

(12) Gabe, E. J.; Le Page, Y.; Charland, J.-P.; Lee, F. L.; White, P. S. J. Appl. Crystallogr. 1989, 22, 384. 
Table 2. Fractional Atomic Coordinates

\begin{tabular}{|c|c|c|c|c|c|c|c|c|c|}
\hline & $x$ & $y$ & $z$ & $B_{\mathrm{eq}}^{a}$ & & $x$ & $y$ & $z$ & $B_{\mathrm{eq}}{ }^{a}$ \\
\hline \multicolumn{10}{|c|}{ (a) $\left[\mathrm{Mo}\left(\mathrm{H}_{2} \mathrm{CPz}_{2}^{\prime}\right)(\mathrm{CO})_{2}(\mathrm{NO})\left(\eta^{\prime}-\mathrm{N}_{3}\right)\right](9)$} \\
\hline Mo & $0.168615(24)$ & $0.99928(3)$ & $0.246872(19)$ & $2.538(14)$ & $C(2)$ & $-0.0109(3)$ & $0.9884(4)$ & $0.16177(24)$ & $3.59(19)$ \\
\hline$N(1)$ & $0.37251(23)$ & $1.02054(24)$ & $0.33196(18)$ & $2.66(14)$ & $C(3)$ & $0.4576(5)$ & $0.8328(4)$ & $0.2874(4)$ & $4.2(3)$ \\
\hline$N(2)$ & $0.42049(24)$ & $1.12594(24)$ & $0.38647(19)$ & $2.78(15)$ & $C(4)$ & $0.4696(3)$ & $0.9548(3)$ & $0.33750(24)$ & $2.94(18)$ \\
\hline$N(3)$ & $0.26674(23)$ & $1.27443(25)$ & $0.30055(20)$ & $2.82(14)$ & $\mathrm{C}(5)$ & $0.5761(3)$ & $1.0171(4)$ & $0.39477(25)$ & $3.55(20)$ \\
\hline $\mathrm{N}(4)$ & $0.18132(24)$ & $1.20373(25)$ & $0.22742(20)$ & $2.77(14)$ & $C(6)$ & $0.5444(3)$ & $1.1249(3)$ & $0.42476(24)$ & $3.16(18)$ \\
\hline $\mathrm{N}(5)$ & $0.13378(21)$ & $1.0074(3)$ & $0.35387(19)$ & $3.20(14)$ & $C(7)$ & $0.6202(5)$ & $1.2277(5)$ & $0.4862(4)$ & $4.8(3)$ \\
\hline$N(6)$ & $0.2098(3)$ & $0.9800(3)$ & $0.11737(19)$ & $3.47(15)$ & $\mathrm{C}(8)$ & $0.3381(4)$ & $1.2177(4)$ & $0.3951(3)$ & $3.14(19)$ \\
\hline$N(7)$ & $0.2816(3)$ & $1.0375(3)$ & $0.09768(20)$ & $3.38(16)$ & $C(9)$ & $0.3511(4)$ & $1.4882(5)$ & $0.3353(4)$ & $4.5(3)$ \\
\hline$N(8)$ & $0.3499(3)$ & $1.0902(4)$ & $0.07512(25)$ & $5.82(22)$ & $\mathrm{C}(10)$ & $0.2670(3)$ & $1.3924(3)$ & $0.2706(3)$ & $3.14(20)$ \\
\hline $\mathrm{O}(1)$ & $0.1386(3)$ & $0.7121(3)$ & $0.2494(3)$ & $7.09(21)$ & $\mathrm{C}(11)$ & $0.1792(3)$ & $1.3985(4)$ & $0.1758(3)$ & $3.50(21)$ \\
\hline$O(2)$ & $-0.11197(23)$ & $0.9752(3)$ & $0.11697(18)$ & $6.04(18)$ & $C(12)$ & $0.1286(3)$ & $1.2816(3)$ & $0.1509(3)$ & $3.18(18)$ \\
\hline$O(3)$ & $0.11261(21)$ & $1.0124(3)$ & $0.42665(17)$ & $5.19(15)$ & $C(13)$ & $0.0297(6)$ & $1.2412(6)$ & $0.0550(4)$ & $4.9(3)$ \\
\hline$C(1)$ & $0.1561(3)$ & $0.8162(4)$ & $0.2508(3)$ & $4.03(21)$ & & & & & \\
\hline \multicolumn{10}{|c|}{ (b) $\left[\mathrm{Mo}\left(\mathrm{H}_{2} \mathrm{CPz}_{2}^{\prime}\right)(\mathrm{CO})(\mathrm{NO})\left(\mathrm{PMe}_{3}\right)_{2}\right] \mathrm{BF}_{4}(\mathbf{1 7})$} \\
\hline Mo & $0.17908(4)$ & $0.99795(7)$ & $0.22662(5)$ & $4.11(3)$ & $O(8)$ & $0.0312(4)$ & $0.9019(6)$ & $0.0920(5)$ & $8.3(4)$ \\
\hline $\mathrm{P}(1)$ & $0.25701(15)$ & $0.82234(19)$ & $0.23852(18)$ & $5.15(14)$ & $\mathrm{C}(11)$ & $0.2534(6)$ & $0.9963(10)$ & $0.4862(6)$ & $8.4(7)$ \\
\hline $\mathrm{P}(2)$ & $0.11155(15)$ & $1.17912(21)$ & $0.21297(21)$ & $6.25(16)$ & $C(12)$ & $0.3099(5)$ & $1.0428(7)$ & $0.4326(5)$ & $5.2(5)$ \\
\hline$N(1)$ & $0.2874(4)$ & $1.0594(5)$ & $0.3408(4)$ & $4.1(3)$ & $\mathrm{C}(13)$ & $0.3916(5)$ & $1.0749(7)$ & $0.4673(5)$ & $5.3(5)$ \\
\hline$N(2)$ & $0.3561(4)$ & $1.1033(5)$ & $0.3168(4)$ & $3.9(3)$ & $C(14)$ & $0.4191(5)$ & $1.1143(6)$ & $0.3934(5)$ & $4.5(4)$ \\
\hline$N(3)$ & $0.2532(4)$ & $1.0467(5)$ & $0.1210(4)$ & $4.2(3)$ & $C(15)$ & $0.4990(5)$ & $1.1634(8)$ & $0.3896(6)$ & $6.2(5)$ \\
\hline $\mathrm{N}(4)$ & $0.3291(4)$ & $1.0940(5)$ & $0.1480(4)$ & $4.1(3)$ & $C(16)$ & $0.1721(6)$ & $0.9647(9)$ & $-0.0265(6)$ & $7.9(7)$ \\
\hline $\mathrm{C}$ & $0.3461(5)$ & $1.1609(6)$ & $0.2308(6)$ & $4.4(4)$ & $C(17)$ & $0.2464(5)$ & $1.0195(7)$ & $0.0308(5)$ & $5.5(5)$ \\
\hline $\mathrm{C}(1)$ & $0.3091(16)$ & $0.7771(14)$ & $0.3419(11)$ & $33.6(24)$ & $C(18)$ & $0.3174(6)$ & $1.0484(8)$ & $0.0026(6)$ & $6.4(6)$ \\
\hline $\mathrm{C}(2)$ & $0.2012(8)$ & $0.7092(10)$ & $0.2021(16)$ & $23.3(18)$ & $C(19)$ & $0.3683(5)$ & $1.0973(7)$ & $0.0766(6)$ & $5.3(5)$ \\
\hline$C(3)$ & $0.3363(11)$ & $0.8102(12)$ & $0.1883(17)$ & $26.7(20)$ & $C(20)$ & $0.4511(6)$ & $1.1462(9)$ & $0.0871(7)$ & $7.6(6)$ \\
\hline$C(4)$ & $0.0032(6)$ & $1.1679(9)$ & $0.2059(11)$ & $13.4(11)$ & B & $0.3892(9)$ & $0.4454(10)$ & $0.2571(9)$ & $7.9(8)$ \\
\hline$C(5)$ & $0.1433(7)$ & $1.2733(9)$ & $0.3069(8)$ & $9.8(8)$ & $\mathrm{F}(1)$ & $0.4082(6)$ & $0.3989(7)$ & $0.3328(4)$ & $18.2(7)$ \\
\hline$C(6)$ & $0.1161(7)$ & $1.2619(9)$ & $0.1142(9)$ & $10.5(8)$ & $F(2)$ & $0.3114(5)$ & $0.4100(7)$ & $0.2199(9)$ & $22.5(9)$ \\
\hline$N(7)$ & $0.1170(5)$ & $0.9571(6)$ & $0.3082(6)$ & $8.1(5)$ & $F(3)$ & $0.4270(6)$ & $0.4042(7)$ & $0.1998(5)$ & $17.8(7)$ \\
\hline$O(7)$ & $0.0694(5)$ & $0.9300(7)$ & $0.3554(6)$ & $11.2(5)$ & $F(4)$ & $0.3791(4)$ & $0.5482(4)$ & $0.2524(5)$ & $12.1(5)$ \\
\hline$C(8)$ & $0.0908(5)$ & $0.9411(6)$ & $0.1398(6)$ & $4.7(4)$ & & & & & \\
\hline \multicolumn{10}{|c|}{ (c) $\left[\mathrm{Mo}\left(\mathrm{Et}_{2} \mathrm{NCS}_{2}\right)(\mathrm{CO})_{2}(\mathrm{NO})\right]_{2}(\mathbf{2 2})$} \\
\hline Mo & $0.47046(3)$ & $0.23606(3)$ & $0.10595(3)$ & $3.163(18)$ & $C(5)^{b}$ & $0.2637(6)$ & $0.6172(8)$ & $0.1250(7)$ & $6.83(23)$ \\
\hline$S(1)$ & $0.38757(8)$ & $0.26866(10)$ & $0.24458(9)$ & $3.14(5)$ & $\mathrm{C}\left(5^{*}\right)^{c}$ & $0.4056(20)$ & $0.6717(25)$ & $0.2778(23)$ & $8.1(8)$ \\
\hline$S(2)$ & $0.42991(9)$ & $0.43926(11)$ & $0.11200(10)$ & $3.52(6)$ & $C(6)$ & $0.5412(3)$ & $0.2410(4)$ & $0.0066(4)$ & $3.99(24)$ \\
\hline $\mathrm{C}(1)$ & $0.3822(3)$ & $0.4102(4)$ & $0.2092(3)$ & $2.99(21)$ & $O(6)$ & $0.5836(3)$ & $0.2423(4)$ & $-0.0530(3)$ & $7.5(3)$ \\
\hline$N(1)$ & $0.34219(25)$ & $0.4840(3)$ & $0.2540(3)$ & $3.54(19)$ & $C(7)$ & $0.4983(3)$ & $0.0753(4)$ & $0.1247(4)$ & $3.74(24)$ \\
\hline $\mathrm{C}(2)$ & $0.3082(4)$ & $0.4576(5)$ & $0.3441(4)$ & $5.0(3)$ & $O(7)$ & $0.5127(3)$ & $-0.0184(3)$ & $0.1311(3)$ & $6.22(23)$ \\
\hline$C(3)$ & $0.2115(4)$ & $0.4624(7)$ & $0.3229(5)$ & $8.2(5)$ & $\mathrm{N}(2)$ & $0.3729(3)$ & $0.1974(4)$ & $0.0038(3)$ & $5.21(25)$ \\
\hline$C(4)$ & $0.3358(4)$ & $0.6021(5)$ & $0.2181(4)$ & $4.9(3)$ & $O(2)$ & $0.3120(3)$ & $0.1702(4)$ & $-0.0620(3)$ & $8.4(3)$ \\
\hline
\end{tabular}

${ }^{a} B_{\text {eq, }}$, equivalent isotropic thermal parameter, is the mean of the principal axes of the thermal ellipsoid. ${ }^{b}$ Mean atoms with occupancy $=0.75$. ${ }^{c}$ Mean atoms with occupancy $=0.25$.

shown. The numbering schemes in the figures correspond to the atomic positions (Table 2). Some selected bond lengths and angles are also collected in Table 3. ORTEP plots for 10 (Figure S1) and 12A (Figure S2), atomic positions for $\mathbf{1 0}$ and 12, more detailed crystal data, a complete table of bond lengths and angles, a table of anisotropic thermal displacement coefficients, and that of hydrogen coordinates of $\mathbf{9 , 1 0}$, 12,17 , and 22 are deposited as supplementary material.

\section{Results and Discussion}

Synthesis of $\left[\mathrm{M}(\mathrm{N}, \mathrm{N})(\mathrm{CO})_{2}(\mathrm{NO})(\mathrm{MeOH})\right] \mathrm{BF}_{4}(\mathrm{M}=\mathrm{Mo}$, $(\mathbf{N}, \mathbf{N})=\mathrm{H}_{2} \mathrm{CPz}_{2}(1), \mathrm{H}_{2} \mathrm{CPz}_{2}{ }_{2}(2) ; \mathrm{M}=\mathrm{W},(\mathbf{N}, \mathbf{N})=\mathrm{H}_{2} \mathrm{CPz}_{2}$ (3), $\mathrm{H}_{2} \mathrm{CPz}_{2}{ }_{2}$ (4)). Complexes $1-4$ are readily formed by nitrosylation of $\left[\mathrm{M}(\mathrm{N}, \mathrm{N})(\mathrm{CO})_{4}\right]$ with $\mathrm{NO}^{+} \mathrm{BF}_{4}{ }^{-}$in $\mathrm{MeOH}$. The structures are believed to be similar to each other, as reflected in the similarity of the IR spectra of the four complexes. From the ${ }^{31} \mathrm{P}\left\{{ }^{1} \mathrm{H}\right\}$ NMR evidence for the reactions between 2 and phosphine ligands and the solid-state structures of $\mathbf{9 , 1 0}$, and 12, described below, the structure for 1-4 may have the nitrosyl group cis to the bidentate ligand, $\mathrm{H}_{2} \mathrm{CPz}_{2}{ }_{2}$, but trans to $\mathrm{MeOH}$ (i.e., $\mathrm{L}_{1}=\mathrm{NO}$ and $\mathrm{L}_{2}=\mathrm{MeOH}$ in Figure 1).

Ligand Substitution of [Mo( $\left.\left.\mathrm{H}_{2} \mathrm{CPz}_{2}^{\prime}\right)(\mathrm{CO})_{2}(\mathrm{NO})(\mathrm{MeOH})\right]$ $\mathbf{B F}_{4}(2)$. The substitution reaction first appeared straightforward, when the weakly coordinated $\mathrm{MeOH}$ of 2 was replaced readily with pyridine (py) to afford $\left[\mathrm{Mo}\left(\mathrm{H}_{2} \mathrm{CPz}_{2}{ }_{2}\right)(\mathrm{CO})_{2}(\mathrm{NO})(\mathrm{py})\right] \mathrm{BF}{ }_{4}$ (5). The reactions soon behaved complicated when some other

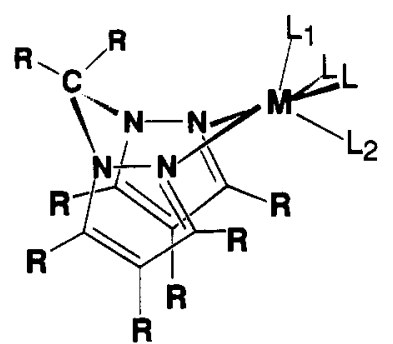

Figure 1. Structural plot of six-coordinate $M(N, N)$ complexes $((N, N)$ $=$ pyrazole-derived bidentate ligands).

nucleophiles were used. A detailed study on the reactions of 2 with many different nucleophiles was hence carried out, and a total of four types of products was observed (Scheme 1). In types 1 and 2 , the reaction products contain $\mathrm{H}_{2} \mathrm{CPz}_{2}{ }_{2}$, whereas, in types 3 and 4 , the products do not have the $\mathrm{H}_{2} \mathrm{CPz}_{2}{ }_{2}$ ligation.

Type 1. Addition of a nitrogen monodentate ligand such as py, oxygen donors such as nitrate and acetate, halides $\left(\mathrm{Cl}^{-}, \mathrm{Br}^{-}\right.$, $\mathrm{I}^{-}$), and pseudohalides (azide, thiocyanide, cyanide) to a solution of 2 in $\mathrm{MeOH}$ afforded 5 and $\left[\mathrm{Mo}\left(\mathrm{H}_{2} \mathrm{CPz}_{2}^{\prime}\right)(\mathrm{CO})_{2}(\mathrm{NO})(\mathrm{L})\right]\left(\mathrm{L}^{-}\right.$ $=\mathrm{Cl}^{-}(6), \mathrm{Br}^{-}(7), \mathrm{I}^{-}(8), \mathrm{N}_{3}^{-}(9), \mathrm{NO}_{3}^{-}(10), \mathrm{SCN}^{-}(11)$, $\left.\mathrm{MeCO}_{2}{ }^{-}(12), \mathrm{CN}^{-}(14)\right)$, respectively. Compound 8 could also be prepared by using Gladfelter's approach ${ }^{13}$ (i.e., by treating the 16-electron compound $\left[\mathrm{Mo}\left(\mathrm{H}_{2} \mathrm{CPz}_{2}{ }_{2}\right)(\mathrm{CO})_{2} \mathrm{Br}_{2}\right]$ with 


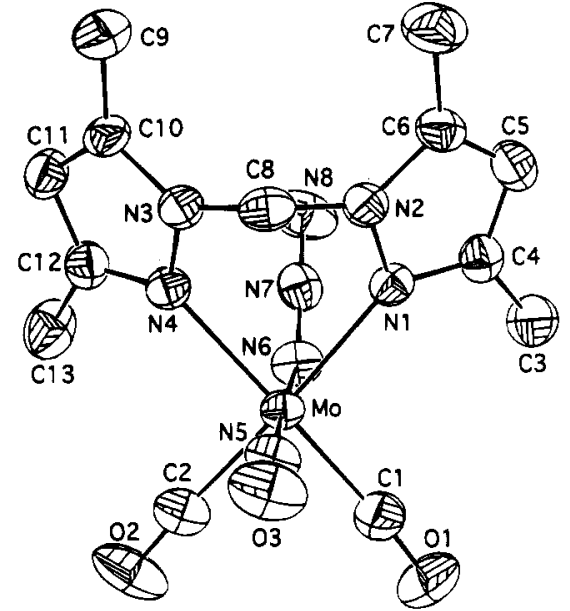

Figure 2. ORTEP plot of $\left[\mathrm{Mo}\left(\mathrm{H}_{2} \mathrm{CPz}_{2}^{\prime}\right)(\mathrm{CO})_{2}(\mathrm{NO})\left(\eta^{\prime}-\mathrm{N}_{3}\right)\right](9)$.

Table 3. Selected Bond Lengths $(\AA)$ and Angles (deg)

\begin{tabular}{llll}
\hline \multicolumn{4}{c}{ (a) $\left[\mathrm{Mo}^{\left.\left(\mathrm{H}_{2} \mathrm{CPz}_{2}^{\prime}\right)(\mathrm{CO})_{2}(\mathrm{NO})\left(\eta^{\prime}-\mathrm{N}_{3}\right)\right](9)}\right.$} \\
$\mathrm{Mo}-\mathrm{N}(1)$ & $2.260(3)$ & $\mathrm{N}(5)-\mathrm{O}(3)$ & $1.199(3)$ \\
$\mathrm{Mo}-\mathrm{N}(4)$ & $2.240(3)$ & $\mathrm{N}(6)-\mathrm{N}(7)$ & $1.190(5)$ \\
$\mathrm{Mo}-\mathrm{N}(5)$ & $1.789(3)$ & $\mathrm{N}(7)-\mathrm{N}(8)$ & $1.151(5)$ \\
$\mathrm{Mo}-\mathrm{N}(6)$ & $2.165(3)$ & $\mathrm{O}(1)-\mathrm{C}(1)$ & $1.141(5)$ \\
$\mathrm{Mo}-\mathrm{C}(1)$ & $1.986(4)$ & $\mathrm{O}(2)-\mathrm{C}(2)$ & $1.129(4)$ \\
$\mathrm{Mo}-\mathrm{C}(2)$ & $2.005(4)$ & & \\
$\mathrm{N}(1)-\mathrm{Mo}-\mathrm{N}(4)$ & $81.73(10)$ & $\mathrm{Mo}-\mathrm{N}(5)-\mathrm{O}(3)$ & $178.81(22)$ \\
$\mathrm{N}(1)-\mathrm{Mo}-\mathrm{C}(2)$ & $175.06(11)$ & $\mathrm{Mo}-\mathrm{N}(6)-\mathrm{N}(7)$ & $127.66(22)$ \\
$\mathrm{N}(6)-\mathrm{N}(7)-\mathrm{N}(8)$ & $177.1(3)$ & $\mathrm{Mo}-\mathrm{C}(1)-\mathrm{O}(1)$ & $174.3(3)$ \\
$\mathrm{N}(4)-\mathrm{Mo}-\mathrm{C}(1)$ & $174.48(13)$ & $\mathrm{Mo}-\mathrm{C}(2)-\mathrm{O}(2)$ & $175.4(3)$ \\
$\mathrm{N}(4)-\mathrm{Mo}-\mathrm{C}(2)$ & $95.11(14)$ & $\mathrm{N}(5)-\mathrm{Mo}-\mathrm{N}(6)$ & $177.27(13)$ \\
$\mathrm{C}(1)-\mathrm{Mo}-\mathrm{C}(2)$ & $83.42(16)$ & &
\end{tabular}

\begin{tabular}{lcll}
\multicolumn{5}{c}{ (b) $\left[\mathrm{Mo}\left(\mathrm{H}_{2} \mathrm{CPz}_{2}^{\prime}\right)(\mathrm{CO})(\mathrm{NO})\left(\mathrm{PMe}_{3}\right)_{2}\right] \mathrm{BF}_{4}(17)$} \\
$\mathrm{Mo}-\mathrm{P}(1)$ & $2.517(3)$ & $\mathrm{C}(8)-\mathrm{O}(8)$ & $1.180(10)$ \\
$\mathrm{Mo}-\mathrm{P}(2)$ & $2.496(3)$ & $\mathrm{P}(1)-\mathrm{C}(2)$ & $1.696(14)$ \\
$\mathrm{Mo}-\mathrm{N}(3)$ & $2.271(6)$ & $\mathrm{P}(1)-\mathrm{C}(3)$ & $1.659(12)$ \\
$\mathrm{Mo}-\mathrm{N}(7)$ & $1.823(8)$ & $\mathrm{P}(2)-\mathrm{C}(4)$ & $1.787(10)$ \\
$\mathrm{Mo}-\mathrm{C}(8)$ & $1.847(8)$ & $\mathrm{P}(2)-\mathrm{C}(5)$ & $1.788(10)$ \\
$\mathrm{P}(1)-\mathrm{C}(1)$ & $1.660(14)$ & $\mathrm{P}(2)-\mathrm{C}(6)$ & $1.789(12)$ \\
$\mathrm{N}(7)-\mathrm{O}(7)$ & $1.211(10)$ & $\mathrm{Mo}-\mathrm{N}(1)$ & $2.290(6)$ \\
$\mathrm{P}(1)-\mathrm{Mo}-\mathrm{P}(2)$ & $175.61(9)$ & $\mathrm{N}(3)-\mathrm{Mo}-\mathrm{C}(8)$ & $95.9(3)$ \\
$\mathrm{N}(3)-\mathrm{Mo}-\mathrm{N}(7)$ & $178.1(3)$ & $\mathrm{N}(7)-\mathrm{Mo}-\mathrm{C}(8)$ & $82.3(4)$ \\
$\mathrm{Mo}-\mathrm{N}(7)-\mathrm{O}(7)$ & $173.9(7)$ & $\mathrm{Mo}-\mathrm{C}(8)-\mathrm{O}(8)$ & $173.2(8)$ \\
$\mathrm{N}(1)-\mathrm{Mo}-\mathrm{N}(3)$ & $87.16(21)$ & $\mathrm{N}(1)-\mathrm{Mo}-\mathrm{N}(7)$ & $94.73(29)$
\end{tabular}

\begin{tabular}{lcll}
\multicolumn{5}{c}{ (c) $\left[\mathrm{Mo}\left(\mathrm{Et}_{2} \mathrm{NCS}_{2}\right)(\mathrm{CO})_{2}(\mathrm{NO})\right]_{2}(22)$} & \\
$\mathrm{Mo}-\mathrm{S}\left(1^{\prime}\right)$ & $2.5549(14)$ & $\mathrm{Mo}-\mathrm{C}(6)$ & $1.937(5)$ \\
$\mathrm{Mo}-\mathrm{S}(1)$ & $2.6326(14)$ & $\mathrm{Mo}-\mathrm{C}(7)$ & $1.984(5)$ \\
$\mathrm{Mo}-\mathrm{S}(2)$ & $2.5306(15)$ & $\mathrm{Mo}-\mathrm{N}(2)$ & $1.844(4)$ \\
$\mathrm{N}(2)-\mathrm{O}(2)$ & $1.176(6)$ & $\mathrm{C}(6)-\mathrm{O}(6)$ & $1.161(6)$ \\
$\mathrm{C}(7)-\mathrm{O}(7)$ & $1.148(6)$ & & \\
$\mathrm{S}(1)-\mathrm{Mo}-\mathrm{S}\left(1^{\prime}\right)$ & $84.07(4)$ & $\mathrm{S}\left(1^{\prime}\right)-\mathrm{Mo}-\mathrm{C}(7)$ & $86.02(14)$ \\
$\mathrm{S}(1)-\mathrm{Mo}-\mathrm{S}(2)$ & $69.66(4)$ & $\mathrm{S}\left(1^{\prime}\right)-\mathrm{Mo}-\mathrm{N}(2)$ & $173.96(16)$ \\
$\mathrm{S}(1)-\mathrm{Mo}-\mathrm{C}(6)$ & $168.76(15)$ & $\mathrm{S}(2)-\mathrm{Mo}-\mathrm{C}(6)$ & $100.50(15)$ \\
$\mathrm{S}(1)-\mathrm{Mo}-\mathrm{C}(7)$ & $100.98(14)$ & $\mathrm{S}(2)-\mathrm{Mo}-\mathrm{C}(7)$ & $170.22(14)$ \\
$\mathrm{S}(1)-\mathrm{Mo}-\mathrm{N}(2)$ & $96.54(15)$ & $\mathrm{S}(2)-\mathrm{Mo}-\mathrm{N}(2)$ & $95.79(15)$ \\
$\mathrm{S}\left(1^{\prime}\right)-\mathrm{Mo}-\mathrm{S}(2)$ & $90.06(4)$ & $\mathrm{C}(6)-\mathrm{Mo}-\mathrm{C}(7)$ & $88.52(20)$ \\
$\mathrm{S}\left(1^{\prime}\right)-\mathrm{Mo}-\mathrm{C}(6)$ & $90.68(16)$ & $\mathrm{Mo}-\mathrm{S}(1)-\mathrm{Mo}{ }^{\prime}$ & $93.31(4)$ \\
$\mathrm{C}(6)-\mathrm{Mo}-\mathrm{N}(2)$ & $89.73(21)$ & $\mathrm{Mo}-\mathrm{C}(6)-\mathrm{O}(6)$ & $179.0(4)$ \\
$\mathrm{C}(7)-\mathrm{Mo}-\mathrm{N}(2)$ & $87.96(21)$ & $\mathrm{Mo}-\mathrm{C}(7)-\mathrm{O}(7)$ & $177.0(4)$ \\
$\mathrm{Mo}-\mathrm{N}(2)-\mathrm{O}(2)$ & $178.1(4)$ & &
\end{tabular}

$\left.\mathrm{M}^{\prime}{ }^{+} \mathrm{NO}_{2}^{-}\left(\mathrm{M}^{\prime+}=\mathrm{Na}^{+}, \mathrm{Ag}^{+}, n-\mathrm{Bu}_{4} \mathrm{~N}^{+}\right)\right)$but with a lower yield. Although compounds 6-12 and 14 display one NO and two $\mathrm{CO}$ stretching bands, compound 5 shows two $\mathrm{NO}$ and two $\mathrm{CO}$ bands in the IR spectra, measured in $\mathrm{CH}_{2} \mathrm{Cl}_{2}$. With the solidstate structures of 9 (Figure 2), 10 (Figure $\mathrm{S1}$ ), and 12 (Figure S2), determined by X-ray crystallography, we believe that 6-12 and 14 all are isostructural with $\mathrm{L}^{-}$cis to $\mathrm{H}_{2} \mathrm{CPz}_{2}{ }_{2}$ but trans to NO. However, in structure 5, there are two different conforma-

(13) Stevens, R. E.; Gladfelter, W. L. Inorg. Chem. 1983, 22, 2034. tions with py cis to $\mathrm{H}_{2} \mathrm{CPz}_{2}{ }_{2}$ but either trans or cis to NO, reflecting the higher $\pi$-acidity of py, compared with $\mathrm{L}^{-}$. In addition, the monohapticity of azide in 9 , nitrate in $\mathbf{1 0}$, and acetate in 12 was also confirmed. The Mo-N distances of $2.224(23)-2.271(24) \AA$ found in 9, 10, and 12 are significantly smaller than those of 2.286(2) and 2.288(2) $\AA$ found in [Mo$\left.\left(\mathrm{H}_{2} \mathrm{CPz}_{2}^{\prime}\right)(\mathrm{CO})_{4}\right]^{14}$ Apparently, a better $\pi$-acid such as NO, compared with $\mathrm{CO}$, can tighten the $\mathrm{H}_{2} \mathrm{CPz}_{2}{ }_{2}$ ligation. However, the $\mathrm{Mo}-\mathrm{N}_{3}$ distance 2.165 (3) $\AA$, the central and terminal $\mathrm{N}-\mathrm{N}$ distances within the $\mathrm{MoN}_{3}$ unit at $1.190(5)$ and $1.151(5) \AA$, respectively, the $\mathrm{Mo}-\mathrm{N}-\mathrm{N}$ angle $127.66(22)^{\circ}$, and the $\mathrm{N}(6)-$ $\mathrm{N}(7)-\mathrm{N}(8)$ angle $177.1(3)^{\circ}$ are found comparable to the reported values. ${ }^{15}$ Apparently, the expected shortening in the $\mathrm{Mo}-\mathrm{N}_{3}$ bond by the electronic tightening effect of the trans-nitrosyl group in 9 is counterbalanced by the increased steric repulsion between the bulky $\mathrm{H}_{2} \mathrm{CPz}_{2}^{\prime}$ and azide groups.

Interestingly, although reaction of 2 with 14 formed an adduct, probably with a cyanide bridge, $\left[\left\{\mathrm{Mo}\left(\mathrm{H}_{2} \mathrm{CPz}_{2}^{\prime}\right)(\mathrm{CO})_{2}(\mathrm{NO})\right\}_{2-}\right.$ $(\mu-\mathrm{CN}) \mathrm{BF}_{4}(13)$, a similar reaction of 2 with 9 or 11 gave no adduct. Obviously, the nucleophilicity of a coordinated azide or thiocyanide ligand decreases appreciably. Although one may deduce from this failure something about the structure of $\mathbf{1 1}$, having a Mo-SCN or Mo-NCS bond, an unambiguous assignment should be subjected to a single-crystal structure analysis or the integrated intensity measurements at $v_{\mathrm{CN}} \cdot{ }^{16 \mathrm{a}}$ The pyridine ligand in 5 was readily replaced by $\mathrm{Cl}^{-}, \mathrm{Br}^{-}$, and $\mathrm{I}^{-}$ in $\mathrm{MeOH}$ to give back 6,7 , and 8 , respectively. However, among these three compounds, only 8 reacted with $\mathrm{NO}^{+} \mathrm{BF}_{4}{ }^{-}$ in $\mathrm{MeOH}$ to reproduce 2. A comparison among the nitrosyl stretching frequencies, displayed by $5-8$ in $\mathrm{CH}_{2} \mathrm{Cl}_{2}$, shows that $v_{\text {No }}$ decreases in the order $5\left(1680\right.$ and $\left.1657 \mathrm{~cm}^{-1}\right)>8(1642$ $\left.\mathrm{cm}^{-1}\right)>7\left(1640 \mathrm{~cm}^{-1}\right)>6\left(1634 \mathrm{~cm}^{-1}\right)$. This order indicates probably that the bonding strength increases as Mo-py $<\mathrm{Mo}-\mathrm{I}$ $<\mathrm{Mo}-\mathrm{Br}<\mathrm{Mo}-\mathrm{Cl}$ in the series of compounds, which may explain the reactivity of 5 toward halides and the result of reaction of 6-8 with $\mathrm{NO}^{+}$. The reaction between 8 and $\mathrm{NO}^{+}$ to give 2 is reminiscent of that between $\mathrm{Et}_{4} \mathrm{~N}\left[\mathrm{Mo}(\mathrm{CO})_{5} \mathrm{I}\right]$ and dppe in the presence of $\mathrm{Et}_{3} \mathrm{OBF}_{4}$ to give $\left[\mathrm{Mo}\left(\eta^{1}\right.\right.$-dppe $\left.)(\mathrm{CO})_{5}\right] .{ }^{17}$ The bonding-strength order appears contradictory to what can be expected, since the iodo ligand is usually regarded as the best or nearly the best $\sigma$-donor. However, it looks reasonable in consideration of the steric effect of the bulky $\mathrm{H}_{2} \mathrm{CPz}_{2}^{\prime}$ ligand, which may inhibit the $\sigma$-donicity by forming a long, weak bond between the sizable $\sigma$-donor and the central metal atom.

Type 2. Although complexes $\left[\mathrm{Mo}\left(\mathrm{H}_{2} \mathrm{CPz}_{2}^{\prime}\right)(\mathrm{CO})_{2}\left(\eta^{3}-\right.\right.$ allyl) $\mathrm{Br}]$ and $\left[\mathrm{Mo}\left(\mathrm{H}_{2} \mathrm{CPz}_{2}{ }_{2}\right)(\mathrm{CO})_{2} \mathrm{Br}_{2}\right]$ were found previously to react with some soft phosphorous monodentate ligands with cone angles less than or equal to $125^{\circ}$ to yield the products without coordination of $\mathrm{H}_{2} \mathrm{CPz}_{2}^{\prime},{ }^{5} 2$ was found to react with any phosphine ligand we tried, including $\mathrm{PPh}_{3}$ (cone angle $\left.145^{\circ}\right),{ }^{18}$ to afford products $\left[\mathrm{Mo}\left(\mathrm{H}_{2} \mathrm{CPz}_{2}^{\prime}\right)(\mathrm{CO})(\mathrm{NO})\left(\mathrm{PR}_{3}\right)_{2}\right] \mathrm{BF}_{4}$ $(\mathrm{R}=\mathrm{Ph}(\mathbf{1 5})$, OMe (16), Me (17)). As shown in the crystal structure of 17 (Figure 3), the two phosphine ligands are cis to $\mathrm{H}_{2} \mathrm{CPz}_{2}^{\prime}$ but trans to each other. Although structure 17 contains

(14) Shiu, K.-B.; Chang, C.-J.; Wang, Y.; Cheng, M.-C. J. Chin. Chem. Soc. (Taipei) 1989, 36, 25.

(15) (a) Pringle, G. E.; Noakes, D. E. Acta Crystallogr. 1968, B24, 262. (b) Young, C. G.; Janos, F.; Bruck, M. A.; Wexler, P. A.; Enemark, J. H. Aust. J. Chem. 1990, 43, 1347. (c) Vicente, R.; Escuer, A.; Ribas, J.; Solans, X. Inorg. Chem. 1992, 31, 1726. (d) Escuer, A.; Vicente, R.; Ribas, J.; Fallah, M. S. E.; Solans, X.; Font-Bardia, M. Inorg. Chem. 1993, 32, 3727.

(16) (a) Kawaguchi, S. Variety in Coordination Modes of Ligands in Metal Complexes; Springer-Verlag: New York, 1988; p 66. (b) Nakamoto, $\mathrm{K}$. Infrared and Raman Spectra of Inorganic and Coordination Compounds, 4th ed.; Wiley: New York, 1986; p 272.

(17) Connor, J. A.; Hudson, G. A. J. Organomet. Chem. 1974, 73, 347. (18) Tolman, C. A. Chem. Rev. 1977, 77, 313. 


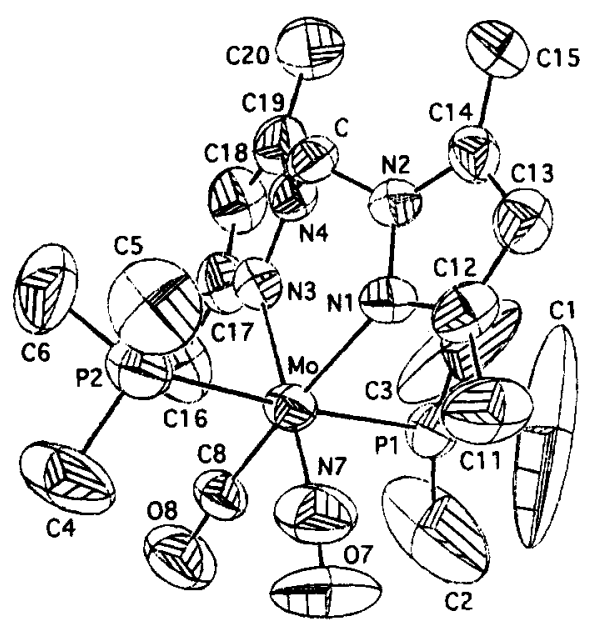

Figure 3. ORTEP plot of the cation, $\left[\mathrm{Mo}\left(\mathrm{H}_{2} \mathrm{CPz}_{2}^{\prime}\right)(\mathrm{CO})(\mathrm{NO})\left(\mathrm{PMe}_{3}\right)_{2}\right]^{+}$ $\left(\mathbf{1 7}^{+}\right)$.

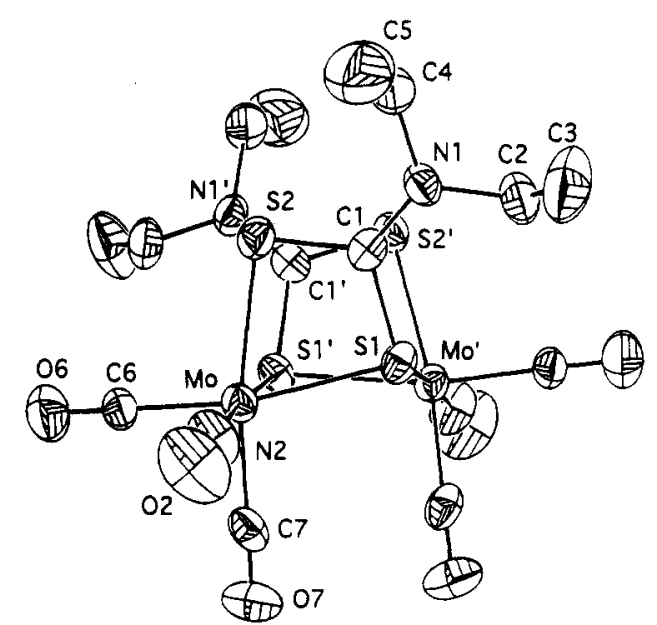

Figure 4. ORTEP plot of $\left[\mathrm{Mo}\left(\mathrm{Et}_{2} \mathrm{NCS}_{2}\right)(\mathrm{CO})_{2}(\mathrm{NO})\right]_{2}(\mathbf{2 2})$.

a bulky boat metallacycle, the Mo-P distances $2.517(3)$ and $2.496(3) \AA$ fall within the reported range $2.369-2.598 \AA .{ }^{19}$ The two significantly different $\mathrm{Mo}-\mathrm{P}$ distances and the angles $\angle \mathrm{P}-$ $\mathrm{Mo}-\mathrm{P}=175.61(9)^{\circ}$, deviating from $180^{\circ}$, indicate that a strong Mo-P bond was achieved via bending the $\mathrm{P}-\mathrm{Mo}-\mathrm{P}$ linkage.

In order to understand a possible reaction pathway for replacing two ligands, $\mathrm{MeOH}$ and $\mathrm{CO}$, of 2 with two $\mathrm{PR}_{3}$ to form 15-17, ${ }^{31} \mathrm{P}\left\{{ }^{1} \mathrm{H}\right\}$ NMR spectra of the reaction mixture of 2 and excess $\mathrm{PR}_{3}$ were measured sequentially in the mixed solvents of $\mathrm{CH}_{2} \mathrm{Cl}_{2}$ and acetone- $d_{6}$ in a ratio of $1 / 1$. Addition of $\mathrm{CH}_{2} \mathrm{Cl}_{2}$ was necessary to help dissolution of 2 and reaction products to form a homogeneous solution, and the deuterated solvent was used for the deuterium lock. After 2 was added to a solution of excess $\mathrm{PMe}_{3}$, three highly intense ${ }^{31} \mathrm{P}$ singlets at

(19) (a) Bush, M. A.; Woodward, P. J. Chem. Soc. A 1968, 1221. (b) Luh, H.; Truter, M. R.; Robson, A. J. Chem. Soc. A 1969, 28. (c) Cheung. K. K.; Lai, T. F.; Lam, S. Y. J. Chem. Soc. A 1970, 3345. (d) Cheung, K. K.; Lai, T. F.; Mok, K. S. J. Chem. Soc. A 1971, 1644. (e) Bridges, D. M.; Holywell, G. C.; Rankin, D. W. H.; Freeman, J. M. J. Organomet. Chem. 1971, 32, 87. (f) Cordes, A. W.; Joyner, R. D.; Shores, R. D.; Dill, E. D. Inorg. Chem. 1974, 13, 132. (g) Sheldrick, W. S. Chem. Ber. 1975, 108, 2242. (h) Sheldrick, W. S. Acto Crystallogr. 1975, B3I, 1789. (i) Sheldrick. W. S. Acta Crystallogr. 1976, B32, 308. (j) De Lerno, J. R.; Trefonas, L. M.; Darensbourg, M. Y.: Majeste, R. J. Inorg. Chem. 1976, 15, 816. (k) Sheldrick, W S.: Bokenstein, A. Acta Crystallogr. 1977, B33, 2916. (1) Johannsen, G.; Stelzer, O. Chem. Ber. 1977, 110, 3438, (m) Atwood, J. L. Darensbourg, D. J. Inorg. Chem. 1977, 16, 2314. (n) Calabrese, J. C. Oakley, R. T.: West, R. Can. J. Chem. 1979, 57, 1909. (o) Gray, G. M.; Fish, F. P.; Srivastava, D. K.; Varshney, A.; Woerd, M. J. van der; Ealick, S. E. J. Organomet. Chem. 1990, 385, 49.
$-61.0,-26.9$, and $-13.0 \mathrm{ppm}$ and one weak broad singlet at $-5.9 \mathrm{ppm}$ were observed. This observation indicates that ligand exchange is not rapid enough to cause chemical shift averaging of complexed and free ligand. The free $\mathrm{PMe}_{3}$ is responsible for the singlet with the greatest intensity at $-61.0 \mathrm{ppm},{ }^{8 \mathrm{a}}$ and to the isolated product 17 belongs the broad peak at $-5.9 \mathrm{ppm}$. According to the theory of Letcher and Van Wazer, ${ }^{20}$ we believe that 2 and $\mathrm{PMe}_{3}$ first give $\left[\mathrm{Mo}\left(\mathrm{H}_{2} \mathrm{CPz}_{2}^{\prime}\right)(\mathrm{CO})_{2}(\mathrm{NO})\left(\mathrm{PMe}_{3}\right)\right] \mathrm{BF}_{4}$ (17-I) with NO trans to $\mathrm{PMe}_{3}\left(\delta\left({ }^{31} \mathrm{P}\right)=-26.9\right)$, and isomerization of this complex soon produces a new structure (17-II) with $\mathrm{CO}$ trans to the phosphine ligand $\left(\delta\left({ }^{31} \mathrm{P}\right)=-13.0\right)$. After $40 \mathrm{~min}$, the signal at $-5.9 \mathrm{ppm}$ became much more intense than the signal at $-61.0 \mathrm{ppm}$, whereas the other two shrank to small signals. This finding is compatible with the brief duration of preparation of 17, monitored by IR measurements, reflecting the strong donating ability of $\mathrm{PMe}_{3}$. However, upon dissolving 2 and excess $\mathrm{PPh}_{3}$ in the solvents, only three singlets appeared at $26.2,34.7$, and -6.0 ppm. After $70 \mathrm{~min}$, the signal at 34.7 ppm increased appreciably but the signal at 26.2 remained almost constant relative to the signal at $-6.0 \mathrm{ppm}$. The singlet at $34.7 \mathrm{ppm}$ was later confirmed to be due to 15 by comparison with the ${ }^{31} \mathrm{P}$ chemical shift of the isolated product $\mathbf{1 5}$ in the solvents. As the singlet at $-6 \mathrm{ppm}$ is due to the free ligand, $\mathrm{PPh}_{3},{ }^{8 \mathrm{~b}}$ the singlet at $26.2 \mathrm{ppm}$ is assigned to either 15-I or 15-II. On the basis of the rapid conversion from 2 to 15 (ca. $2 \mathrm{~h}$ ) and the almost constant intensity observed for this singlet relative to the $\mathrm{PPh}_{3}$ peak, we assign the singlet to 15-I. Probably the bulky $\mathrm{PPh}_{3}$ may help induce decarbonylation sterically so that 15-II reacted with $\mathrm{PPh}_{3}$ with loss of one $\mathrm{CO}$ as soon as it was formed from 15-I to give 15 . Unlike the reaction between 2 and $\mathrm{PPh}_{3}$, that between 2 and $\mathrm{P}(\mathrm{OMe})_{3}$ gave four singlets at $\delta 152.2$ (for 16), 29.9 (for 16-I), 54.5 (for 16-II), and $141 \mathrm{ppm}$ (for free $\left.\mathrm{P}(\mathrm{OMe})_{3}\right){ }^{8 \mathrm{a}}$

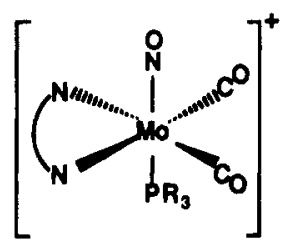

15- 16- or 17-1

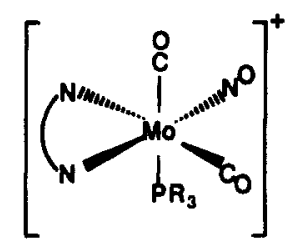

15-, 16- or 17-11
Type 3. Soft neutral bidentate ligands (L,L) were found to convert 2 into $c i s-\left[\mathrm{Mo}(\mathrm{L}, \mathrm{L})_{2}(\mathrm{CO})(\mathrm{NO})\right] \mathrm{BF}_{4}((\mathrm{~L}, \mathrm{~L})=$ bpy $(\mathbf{1 8})$, phen (19), $\mathrm{Me}_{2}$ bpy (20), dppm (21)). The reaction products of 2 with dppm depend on the solvent used. Upon mixing 2 and dppm in a mixed solvent system of $\mathrm{CH}_{2} \mathrm{Cl}_{2}$ and acetone- $d_{6}$ in a ratio of $1 / 1$, one singlet at $-22.3 \mathrm{ppm}$ (for free dppm), ${ }^{8 c}$ four clear doublets, and two apparent triplets appeared (Figure 5a). The doublets at 22.1 and $-26.0 \mathrm{ppm}$ with $J=110 \mathrm{~Hz}$ are assigned to coordinated and free phosphorus atoms of $\eta^{1}$-dppm, respectively, in $21-\mathrm{I}$ and those at 30.2 and $-28.9 \mathrm{ppm}$ with $J$ $=52 \mathrm{~Hz}$ are assigned to 21-II. After spectrum expansion, the triplets were decomposed into four doublets at 29.1 and -27.1 ppm with $J=26.3 \mathrm{~Hz}$ and 29.3 and $-27.3 \mathrm{ppm}$ with $J=26.0$ $\mathrm{Hz}$. These four doublets are assigned to $\left[\mathrm{Mo}\left(\mathrm{H}_{2} \mathrm{CPz}_{2}^{\prime}\right)\right.$ $\left(\eta^{1} \text {-dppm }\right)_{2}(\mathrm{CO})(\mathrm{NO}) \mathrm{BBF}_{4}($ (21-III) with one dppm trans to another dppm, which is expected to have a structure similar to that of 17 (Figure 3). The ring-flip motion of the six-memberedboat metallacycle does not occur in solution for $15,16,17$, or 21-III to average the phosphorus chemcial shifts; hence two closed doublets at the high fields and two at the low fields are observed for 21-III whereas two closed singlets overlap as a unresolved broad peak found for 15, 16, and 17. After $90 \mathrm{~min}$,

(20) Letcher, J. H.; Van Wazer, J. R. J. Chem. Phys. 1966, 44, 815. 

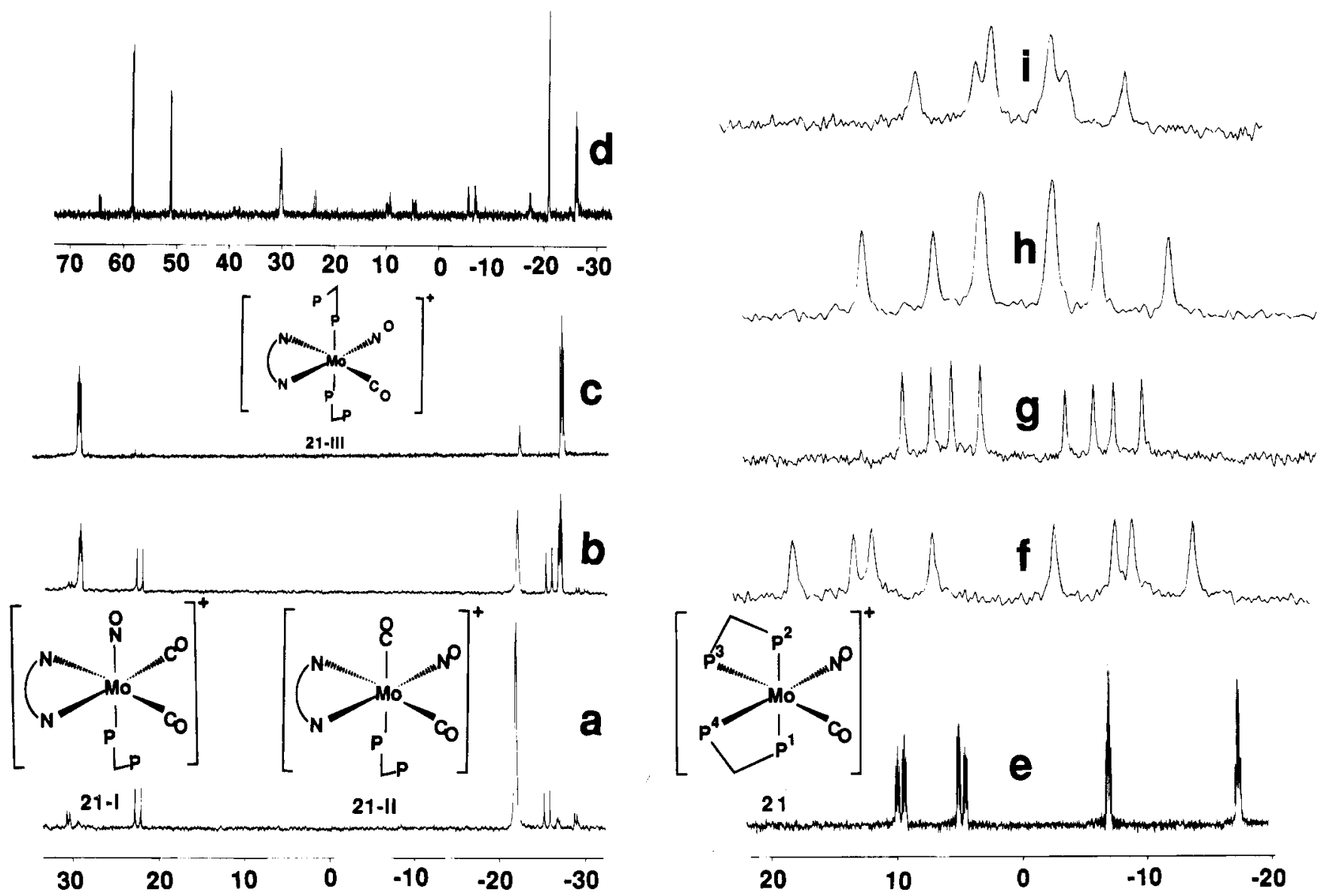

Figure 5. The $162-\mathrm{MHz}^{31} \mathrm{P}\left\{{ }^{1} \mathrm{H}\right\}$ NMR spectra recorded for (a) $\left.\left[\mathrm{Mo}_{(} \mathrm{H}_{2} \mathrm{CPz}^{\prime}{ }_{2}\right)(\mathrm{CO})_{2}(\mathrm{NO})(\mathrm{MeOH})\right] \mathrm{BF}_{4}$ (2) and dppm at $t=0$; (b) at $t=90 \mathrm{~min}$; (c) 2 and excess dppm at $t=60 \mathrm{~min}$; (d) 2 and excess dppm at $t=70 \mathrm{~h}$ and (e) cis-[Mo(dppm) $\left.)_{2}(\mathrm{CO})(\mathrm{NO})\right] \mathrm{BF}_{4}(21)$. The expanded ${ }^{31} \mathrm{P}\left\{{ }^{1} \mathrm{H}\right\}$ spectrum of 21 at (f) $\delta^{31} \mathrm{P}=9.54 ;(\mathrm{g}) \delta^{31} \mathrm{P}=4.63$; (h) $\delta^{31} \mathrm{P}=-7.25$ and (i) $\delta{ }^{31} \mathrm{P}=-17.75$.

the triplets (of 21-III) increased appreciably (Figure 5b). Adding further dppm brought the complete conversion of 2 into 21-III (Figure 5c). Alternatively, we added in about $1 \mathrm{~h}$ a solution of $2(1.0 \mathrm{mmol})$ in $\mathrm{CH}_{2} \mathrm{Cl}_{2}(25 \mathrm{~mL})$ dropwise to another solution of dppm $(3.0 \mathrm{mmol})$ in $\mathrm{CH}_{2} \mathrm{Cl}_{2}(25 \mathrm{~mL})$ to form a solution of 21-III with excess dppm, giving a ${ }^{31} \mathrm{P}\left\{{ }^{1} \mathrm{H}\right\}$ NMR spectrum similar to Figure 5c. However, the color of this solution gradually altered from orange-red into dark brown after $23 \mathrm{~h}$. After $70 \mathrm{~h}$, an IR solution spectrum showed "completion" of the reaction; it contains two $v_{\mathrm{CO}}$ bands at $1944 \mathrm{~m}$ and 1917 $\mathrm{s} \mathrm{cm}{ }^{-1}$ and two $v_{\mathrm{NO}}$ bands at $1617 \mathrm{~s}$ and $1597 \mathrm{sh} \mathrm{cm}^{-1}$. A ${ }^{31} \mathrm{P}\left\{{ }^{1} \mathrm{H}\right\}$ NMR spectrum was recorded for this solution and consisted of many lines, located at the range +64 to $-27 \mathrm{ppm}$ (Figure 5d). Although we could not separate various products from this reaction on a column chromatograph, we noticed that at least one product did not dissolve in $\mathrm{MeOH}$. Accordingly, a mixture of $2(0.45 \mathrm{mmol})$ and dppm $(1.00 \mathrm{mmol})$ in $\mathrm{MeOH}$ $\left(20 \mathrm{~mL}\right.$ ) was heated at $65^{\circ} \mathrm{C}$ for $2 \mathrm{~h}$. A pure compound (21) was obtained as precipitate in satisfactory yield. The ${ }^{31} \mathrm{P}\left\{{ }^{1} \mathrm{H}\right\}$ NMR spectrum of 21 contains four multiplets (Figure 5e), and the ${ }^{1} \mathrm{H}\left\{{ }^{31} \mathrm{P}\right\}$ NMR spectrum of this compound has four doublets at $4.71,4.44,4.35$, and $3.82 \mathrm{ppm}$ with $J=15 \mathrm{~Hz}$, consistent with a cis disposition of two $\eta^{2}$-dppm ligands in 21 . When the ${ }^{31} \mathrm{P}\left\{{ }^{1} \mathrm{H}\right\}$ NMR spectrum was recorded without NOE, the four multiplets were found in an integration ratio 1.00:1.05:1.04: 1.01. These multiplets are assigned to the four $P$ atoms shown in Figure 5e, based on various coupling parameters and the theory of Letcher and Van Wazer. ${ }^{20}$ The doublet of doublet of doublet at $9.54 \mathrm{ppm}(J=21,27,88 \mathrm{~Hz})$ is assigned to $\mathrm{P}^{1}$ (Figure $5 \mathrm{f}$ ), and another doublet of doublet of doublet at $4.63 \mathrm{ppm}(J$
$=16,27,88 \mathrm{~Hz}$ ) is assigned to $\mathrm{P}^{2}$ (Figure $5 \mathrm{~g}$ ); the doublet of triplet at $-7.25 \mathrm{ppm}(J=16,27 \mathrm{~Hz})$ is assigned to $\mathrm{P}^{3}$ (Figure $5 \mathrm{~h})$, and another doublet of triplet at $-17.75 \mathrm{ppm}(J=21,27$ $\mathrm{Hz}$ ) is assigned to $\mathrm{P}^{4}$ (Figure 5i). Hence, $J\left(\mathrm{P}^{1}, \mathrm{P}^{2}\right)=88, J\left(\mathrm{P}^{1}\right.$, $\left.\mathrm{P}^{3}\right)=J\left(\mathrm{P}^{2}, \mathrm{P}^{4}\right)=J\left(\mathrm{P}^{3}, \mathrm{P}^{4}\right)=27, J\left(\mathrm{P}^{1}, \mathrm{P}^{4}\right)=21$, and $J\left(\mathrm{P}^{2}, \mathrm{P}^{3}\right)$ $=16 \mathrm{~Hz}$. The signals due to $\mathrm{P}^{4}$, trans to NO, are broadened, corroborating the assignment of that particular atom. ${ }^{21}$ According to detailed reports about metal complexes of dppm, ${ }^{22}$ the lines at positions greater than $40 \mathrm{ppm}$, shown in Figure 5d, are possibly due to formation of multinuclear complexes containing $\mu$-dppm linkages, such as $\left\{\mathrm{Mo}_{2}(\mu \text {-dppm })_{2}\right\}^{2+}$ complexes.

Unlike the reaction of 2 with dppm, those of 2 with bpy, phen, and $\mathrm{Me}_{2}$ bpy are straightforward. In either $\mathrm{CH}_{2} \mathrm{Cl}_{2}$ or $\mathrm{MeOH}$, only one product was obtained to give 18,19 , and 20 , respectively. As no further substituted product such as [Mo$\left(\mathrm{H}_{2} \mathrm{CPz}_{2}^{\prime}\right)(\mathrm{py})_{2}(\mathrm{CO})(\mathrm{NO}) \mathrm{BF}_{4}$ was formed from the reaction of 5 with excess pyridine in $\mathrm{MeCN}$ under forcing conditions, the reaction pathway from 2 to $18-20$ may involve two successive intermediates such as $\left[\mathrm{Mo}\left(\mathrm{H}_{2} \mathrm{CPz}_{2}^{\prime}\right)\left(\eta^{\prime}\right.\right.$-bpy $\left.)(\mathrm{CO})_{2}(\mathrm{NO})\right] \mathrm{BF}_{4}$ and

(21) Connelly, N. G.; Gardner, C. J. Chem. Soc., Dalton Trans. 1979, 609.

(22) (a) Balch. A. L. In Homogeneous Catalysis with Metal Phosphine Complexes; Pignolet, L. H., Ed.; Plenum Press: New York, 1983; p 167. (b) Puddephatt, R. J. Chem. Soc. Rev. 1983, 99. (c) Chaudret, B.; Delavaux, B.; Poiblanc, R. Coord. Chem. Rev. 1988, 86, 191. (d) Manojlovic-Muir, L.; Mirza, H. A.; Sadiq, N.; Pudephatt, R. J. Inorg. Chem. 1993, 32, 117, (e) Braunstein, P.; Bellefon, C. M.; Oswald, B.; Ries, M.; Lanfranchi, M.; Tiripicchio, A. Inorg. Chem. 1993, 32, 1638. (f) Braunstein, P.; Bellefon, C. M.; Oswald, B. Inorg. Chem. 1993, 32, 1649. (g) Balch, A. L.; Noll, B. C.; Olmstead, M. M.; Toronton, D. V. Inorg. Chem. 1993, 32, 3613. 
Table 4. Angles $\alpha, \beta, \gamma$, and $\theta$ for (N,N)-Ligated Complexes

\begin{tabular}{|c|c|c|c|c|c|}
\hline complex ${ }^{a}$ & $\alpha$ & $\beta$ & $\gamma$ & $\theta$ & ref \\
\hline$\left[\mathrm{W}\left(\mathrm{H}_{2} \mathrm{CPz}_{2}^{\prime}\right)(\mathrm{CO})_{4}\right]$ & $111.8(3)$ & $146.6(3)$ & $128.4(7)$ & $167.7(3)$ & $9 \mathrm{~b}$ \\
\hline$\left[\mathrm{W}\left(\mathrm{H}_{2} \mathrm{CPz}_{2}{ }_{2}\right)(\mathrm{CO})_{4}\right]$ & $120.8(3)$ & $152.1(2)$ & $125.0(5)$ & $170.8(2)$ & $9 \mathrm{~b}$ \\
\hline$\left[\mathrm{Mo}\left(\mathrm{H}_{2} \mathrm{CPz}_{2}^{\prime}\right)(\mathrm{CO})_{4}\right]$ & $111.9(2)$ & $147.2(2)$ & $126.7(2)$ & $167.3(1)$ & 14 \\
\hline$\left[\mathrm{Mo}\left(\mathrm{PhHCPz}_{2}^{\prime}\right)(\mathrm{CO})_{4}\right]$ & $127.6(4)$ & $159.7(4)$ & $128.7(4)$ & $166.0(2)$ & 27 \\
\hline \multirow[t]{2}{*}[\mathrm{Mo}\{\mathrm{H}_{2}\mathrm{C}(\mathrm{Pz}^{*})(\mathrm{Pz}^{*})\}(\mathrm{CO})_{4}]{$^{b}$} & $111.4(3)$ & $148.4(3)$ & $127.1(6)$ & $165.7(3)$ & 27 \\
\hline & $105.2(3)$ & 146.3(3) & $127.4(6)$ & $164.6(3)$ & 27 \\
\hline$\left[\mathrm{Mo}\left\{\mathrm{H}_{2} \mathrm{C}\left(\mathrm{Pz}^{\ddagger}\right)_{2}\right\}(\mathrm{CO})_{4}\right]$ & $130.1(3)$ & $150.5(2)$ & $123.9(6)$ & $165.9(3)$ & 27 \\
\hline$\left[\mathrm{Mo}\left(\mathrm{H}_{2} \mathrm{CPz}_{2}^{\prime}\right)(\mathrm{CO})_{2}(\mathrm{NO})\left(\mathrm{N}_{3}\right)\right]$ & $119.4(2)$ & $150.6(1)$ & $124.6(4)$ & $177.3(1)$ & this work \\
\hline \multirow[t]{2}{*}[\mathrm{Mo}(\mathrm{H}_{2}\mathrm{CPz}_{2}^{\prime})(\mathrm{CO})_{2}(\mathrm{NO})(\mathrm{MeCO}_{2})]{$\mathrm{BF}_{4}^{b}$} & $121.6(1)$ & $150.9(1)$ & $127(3)$ & $177.6(1)$ & this work \\
\hline & $120.8(1)$ & $152.9(1)$ & $129(3)$ & $176.4(1)$ & this work \\
\hline$\left[\mathrm{Mo}\left(\mathrm{H}_{2} \mathrm{CPz}^{\prime}{ }_{2}\right)(\mathrm{CO})_{2}(\mathrm{NO})\left(\mathrm{NO}_{3}\right)\right]$ & $122.3(2)$ & $155.5(2)$ & $123.6(3)$ & $177.3(1)$ & this work \\
\hline$\left[\mathrm{Mo}\left(\mathrm{H}_{2} \mathrm{CPz}_{2}^{\prime}\right)(\mathrm{CO})(\mathrm{NO})\left(\mathrm{PMe}_{3}\right)_{2}\right] \mathrm{BF}_{4}$ & $153.9(4)$ & 179.1(3) & $119.9(8)$ & $175.6(9)$ & this work \\
\hline
\end{tabular}

${ }^{a} \mathrm{Pz}^{\prime \prime}=$ 3,4,5-trimethylpyrazol-1-yl; $\mathrm{Pz}^{*}=$ 3-phenylpyrazol-1-yl; $\mathrm{Pz}^{* \prime}=5$-phenylpyrazol-1-yl; $\mathrm{Pz}^{\ddagger}=$ 3,5-dimethyl-4-benzylpyrazol-1-yl. ${ }^{b}$ Two different molecules in the asymmetric unit of the crystal used.

$\left[\mathrm{Mo}\left(\eta^{2}\right.\right.$-bpy $)\left(\eta^{1}\right.$-bpy $\left.)(\mathrm{CO})_{2}(\mathrm{NO})\right] \mathrm{BF}_{4},{ }^{23}$ the latter of which then formed the final product 18. The cis arrangement of two nitrogen bidentate ligands is clearly supported by observation of four methyl singlets for 20 in a ${ }^{1} \mathrm{H}$ NMR spectrum. The compound of $c i s-\left[\mathrm{Mo}(\mathrm{bpy})_{2}(\mathrm{CO})(\mathrm{NO})\right] \mathrm{PF}_{6}$ was reported in 1984 , from the reaction of $c i s-\left[\mathrm{Mo}(\mathrm{CO})_{2}(\mathrm{bpy})_{2}\right]$ with $\mathrm{NO}^{+} \mathrm{PF}_{6}-{ }^{24}$ Spectral data of this compound and $\mathbf{1 8}$ are similar. However, caution in using an appropriate proportion of $\mathrm{NO}^{+} \mathrm{PF}_{6}{ }^{-}$to react with cis-[Mo(CO $\left.)_{2}(\mathrm{bpy})_{2}\right]$ was described for preparation of cis$\left[\mathrm{Mo}(\mathrm{bpy})_{2}(\mathrm{CO})(\mathrm{NO})\right] \mathrm{PF}_{6}$, because excess nitrosylating agent produced only an intractable oily material. The influence of strongly donating $\mathrm{N}-\mathrm{N}$ ligands $(\mathrm{N}-\mathrm{N}=$ bpy and phen), permitting access of cis-[M(N-N $\left.)_{2}(\mathrm{CO})_{2}\right]$ to $c i s-\left[\mathrm{M}(\mathrm{N}-\mathrm{N})_{2}-\right.$ $\left.(\mathrm{CO})_{2}\right]^{2+}$ and $c i s-\left[\mathrm{M}(\mathrm{N}-\mathrm{N})_{2}(\mathrm{CO})_{2} \text { (solvent) }\right]^{2+}$, was also described. ${ }^{24}$ Coincidentally, Bond and co-workers found previously that cis-[M(dppm $\left.)_{2}(\mathrm{CO})_{2}\right]$ compounds are oxidized and isomerized easily. ${ }^{25}$ This propensity would make preparation of 21 difficult via nitrosylation of $c i s-\left[\mathrm{M}(\mathrm{dppm})_{2}(\mathrm{CO})_{2}\right]$ with $\mathrm{NO}^{+} \mathrm{PF}_{6}{ }^{-}$. The reactions of 2 (and 4 ) with either nitrogen or phosphorus bidentate ligands (L,L) to give cis-[M(L,L) $)_{2}(\mathrm{CO})$ (NO) $\mathrm{BF}_{4}$ such as $18,19,20$, or 21 may represent a more convenient synthetic method.

Type 4. The reaction of 2 with dithiocarbamate anion, $(\mathrm{S}, \mathrm{S})^{-}$, proceeded smoothly according to the solution IR spectra. However, after removal of solvent, there appeared one orangered compound $\left[\mathrm{Mo}(\mathrm{S}, \mathbf{S})(\mathrm{CO})_{2}(\mathrm{NO})\right]_{2}\left((\mathrm{~S}, \mathbf{S})^{-}=\mathrm{Et}_{2} \mathrm{NCS}_{2}{ }^{-}{ }^{-22}\right)$, $\mathrm{Me}_{2} \mathrm{NCS}_{2}^{-}$(23), $\left.\mathrm{C}_{4} \mathrm{H}_{8} \mathrm{NCS}_{2}{ }^{-}(24)\right)$ as major product and one yellow compound as minor product. Only the orange-red compound was cleanly separated and characterized. Dinuclearity of compounds associated with the six-electron dithiocarbamato ligand in a $\mu, \eta^{2}$-fashion is confirmed by the structural determination of 22 (Figure 4), which contains a crystallographically imposed inversion center. The distance of 3.474(3) $\AA$ between two $S$ atoms and that of 3.773(1) $\AA$ between two Mo atoms is compatible with no S-S or Mo-Mo bond. The nitrosyl groups are trans to the bridging sulfur atoms while cis to carbonyls. The failure to isolate and to characterize the yellow product causes ambiguity about the possible pathway to form $\left[\mathrm{Mo}(\mathrm{S}, \mathrm{S})(\mathrm{CO})_{2}(\mathrm{NO})\right]_{2}$ from 2 . However, on the basis of the bridging propensity of organic sulfur compounds, ${ }^{26}$ the

(23) Cao, S.; Reddy, K. B.; Eyring, E. M.; van Eldik, R. Organometallics 1994, 13, 91 .

(24) Connor, J. A.; James, E. J.; Overton, C.; Murr, N. E. J. Chem. Soc., Dalton Trans. 1984, 255.

(25) Bond, A. M.; Colton, R.; Jackowski, J. J. Inorg. Chem. 1975, 14, 274, 2526.

(26) (a) Vahrenkamp, H. Angew. Chem., Int. Ed. Engl. 1975, 14, 322. (b) Lu, Z.; White, C.; Rheingold, A. L.; Crabtree, R. H. Inorg. Chem. $1993,32,3991$.

(27) Shiu, K.-B.; Lin, S.-T.; Chou, C.-C.; Peng, S.-M.; Cheng, M.-C.; Wang, S.-L.; Liao, F.-L. J. Organomet. Chem. 1994, 469, 169. pathway may involve an intermediate like $\left[\mathrm{Mo}\left(\mathrm{H}_{2} \mathrm{CPz}_{2}^{\prime}\right)\left(\eta^{1}\right.\right.$ $\left.(\mathrm{S}, \mathrm{S}))(\mathrm{CO})_{2}(\mathrm{NO})\right]$, which loses $\mathrm{H}_{2} \mathrm{CPz}_{2}{ }_{2}$ and forms the $16-$ electron compound $\left[\mathrm{Mo}\left(\eta^{2}-(\mathrm{S}, \mathrm{S})\right)(\mathrm{CO})_{2}(\mathrm{NO})\right]$. Dimerization of this intermediate then affords the product $\left[\mathrm{Mo}(\mathrm{S}, \mathrm{S})(\mathrm{CO})_{2}(\mathrm{NO})\right]_{2}$.

Conformational Difference in the Six-Membered Boat Metallacycles. Previously, we reported that the conformation of the six-membered metallacycle, formed by a $(\mathrm{N}, \mathrm{N})$ ligand and a metal atom, is flexible, depending on the substituents on the pyrazolyl skeleton. ${ }^{9 b}$ We now wish to add that different ligands in the coordination sphere of a metal atom can also cause a different conformation of the metallacycle. Though the crystallographic data refer to a molecule in a crystal field and undergoing vibrational motion, various metallacycle conformations present in $M(N, N)$ solid-state structures reflect that the complexes may retain the metallacycle flexibility in solution or during a reaction course. ${ }^{28}$ In order to gain a better understanding about this flexible metallacycle, we calculated four angles $\alpha, \beta, \gamma$, and $\theta$ of all $\mathrm{M}(\mathrm{N}, \mathrm{N})$ structures, including 9, 10, 12, and 17 (Table 4). Angle $\alpha$ is defined by the two azolyl planes, angle $\beta$ by the MNN and the NNNN planes, angle $\gamma$ by the NNNN and the CNN planes, and angle $\theta$ is formed by the two ligands, trans to each other but cis to $\mathrm{H}_{2} \mathrm{CPz}_{2}^{\prime}$ (i.e., $\angle \mathrm{L}_{1}-\mathrm{M}-\mathrm{L}_{2}=\theta$; cf. Figure 1). To make a consistent set of $\alpha, \beta$, and $\gamma$ values, we should also calculate angle $\omega$, defined by the MNN and the CNN planes, and two vertical distances from the boat-end atom, $\mathrm{Mo}$ (or $\mathrm{C}$ ), to each azolyl plane. Simple geometry indicates that the angles $\beta, \gamma$, and $\omega$ should follow $\beta$ $+\gamma-180^{\circ}=\omega$; if one distance is positive, the other value should be negative.

By comparison of the four angles of $M(N, N)$ complexes (Table 4), it is quite clear that the metallacyles in nitrosyl complexes are more distorted than those in $\left[\mathrm{M}(\mathrm{N}, \mathrm{N})(\mathrm{CO})_{4}\right]$ complexes. The range of angles found for $\alpha, \beta$, or $\gamma$ is larger in the nitrosyl-ligated complexes $\left(\Delta \alpha=\alpha_{\max }-\alpha_{\min }=34.5^{\circ}\right.$; $\left.\Delta \beta=28.5^{\circ} ; \Delta \gamma=9.1^{\circ}\right)$ than that in $\left[\mathrm{M}(\mathrm{N}, \mathrm{N})(\mathrm{CO})_{4}\right]$ complexes $\left(\Delta \alpha=24.9^{\circ} ; \Delta \beta=13.4^{\circ} ; \Delta \gamma=4.8^{\circ}\right)$. The $\theta$ values found in $\left[\mathrm{M}(\mathrm{N}, \mathrm{N})(\mathrm{CO})_{4}\right]$ complexes are $164.6-170.8^{\circ}$, much smaller than those of $175.6-177.6^{\circ}$ in the nitrosyl complexes. In either complex, the feature of $\beta>\gamma$ may reflect the much greater congestion near the metal center.

As already mentioned above, the fact that the $\mathrm{Mo}-\mathrm{N}$ distances $2.224(23)-2.271(24) \AA$ found in the nitrosyl complexes 9,10 , and 12 are significantly shorter than those of $2.286(2)$ and $2.288(2) \AA$ found in $\left[\mathrm{Mo}\left(\mathrm{H}_{2} \mathrm{CPz}_{2}^{\prime}\right)(\mathrm{CO})_{4}\right]^{14}$ reflects the presence of the electronic tightening effect of a nitrosyl group. It thus appears obvious that this effect can induce a more distorted structure in the nitrosyl-ligated complexes by shortening the $\mathrm{Mo}-\mathrm{N}$ bonds.

(28) Orpen, A. G. Chem. Soc. Rev. 1993, 22, 191. 


\section{Conclusions}

The present study demonstrates that the presence of a nitrosyl group in a complex such as 2 can help to tighten the $\mathrm{H}_{2} \mathrm{CPz}_{2}$ ligation during some nucleophilic substitution reactions in spite of the inherent ring strain present in the six-membered-boat metallacycle, formed between $\mathrm{H}_{2} \mathrm{CPz}_{2}{ }_{2}$ and the central metal atom (Figure 1). Flexibility of the metallacycle is still present even with this electronic tightening in all the $\mathrm{H}_{2} \mathrm{CPz}_{2}{ }_{2}$-ligated metal nitrosyl products (Table 4). It appears that the steric effect of $\mathrm{H}_{2} \mathrm{CPz}_{2}^{\prime}$, the inherent ring strain and flexibility of the metallacyle, and the electronic effect of $\mathrm{NO}$ contribute to allow formation of four types of products from the reactions of 2 (Scheme 1). Clearly, the results of this study establish benchmarks for future studies on the stereochemical effect of $(\mathrm{N}, \mathrm{N})$ in the related $\mathrm{M}(\mathrm{N}, \mathrm{N})$ complexes.

Acknowledgment. We wish to thank the National Science Council of the Republic of China for the financial support of this research (Contract No. NSC83-0208-M006-35) and Mr. Yao-Tin Wu for his skilled technical assistance.

Supplementary Material Available: ORTEP plots of 10 (Figure S1) and 12 (Figure S2), atomic positions for 10 and 12 (Table S1), complete crystal data (Table S2), bond lengths and angles (Table S3), anisotropic thermal displacement coefficients of non-hydrogen atoms (Table S4), and fractional atomic coordinates for hydrogen atoms in 9 , 10, 12, 17, and 22 (Table S5) (18 pages). Ordering information is given on any current masthead page.

IC9406401 